\title{
Abstracts From the December 2018 International Sports and Exercise Nutrition Conference in Newscastle upon Tyne
}

\section{Day 1- Orals: Performance Theme}

Protein Supplementation Does Not Further Augment Physiological Adaptations to Prolonged Endurance Exercise Training

KL Jonvik ${ }^{1}$, KJM Paulussen ${ }^{1}$, SL Danen ${ }^{1}$, AMH Horstman ${ }^{3}$, FC Wardenaar $^{1,4}$, LJC van Loon ${ }^{1,2}$, and JW van Dijk ${ }^{1}$

${ }^{1}$ HAN University of Applied Sciences, Nijmegen, the Netherlands

${ }^{2}$ Maastricht University Medical Centre+, Maastricht, the Netherlands

${ }^{3}$ FrieslandCampina, Amersfoort, the Netherlands

${ }^{4}$ Arizona State University, Phoenix, Arizona, USA

It has recently been speculated that protein supplementation may further augment the adaptations to prolonged endurance exercise training. We assessed the impact of protein supplementation during prolonged endurance exercise training on whole-body oxidative capacity $\left(\mathrm{VO}_{2 \max }\right)$ and endurance exercise performance. Sixty recreationally active males (age: $27 \pm 6$ y; BMI: $23.8 \pm 2.6 \mathrm{~kg} \cdot \mathrm{m}^{-2}, \mathrm{VO}_{2 \max } 47 \pm 6 \mathrm{~mL} \cdot \mathrm{min}^{-1} \cdot \mathrm{kg}^{-1}$ ) were subjected to 12 weeks of triweekly endurance exercise training. After each session and each night prior to sleep, participants ingested either a protein supplement (PRO; $29 \mathrm{~g}$ casein protein) or an isocaloric carbohydrate placebo (PLA). Before and after the 12 weeks of training, $\mathrm{VO}_{2 \max }$ and endurance exercise performance $(\sim 10-\mathrm{km}$ time-trial) were assessed on a cycle ergometer. Muscular endurance (total workload achieved during 30 reciprocal isokinetic contractions) was assessed by isokinetic dynamometry and body composition by DXA. Dietary intake was assessed at baseline and during the intervention period. Repeated measures ANOVA was applied to assess whether training adaptations were different between groups. Protein intake increased in PRO $\left(1.2 \pm 0.4\right.$ to $\left.1.6 \pm 0.3 \mathrm{~g} \cdot \mathrm{kg}^{-1}\right)$, but not in PLA $(1.3 \pm 0.4$ to $1.2 \pm 0.3 \mathrm{~g} \cdot \mathrm{kg}^{-1}$, time $\mathrm{x}$ treatment interaction, $\left.P<0.001\right)$. Endurance exercise training induced an $11 \pm 6 \%$ increase in $\mathrm{VO}_{2 \max }$ (time effect, $P<0.001$ ), with no differences between groups (PRO: $48 \pm 6$ to $53 \pm 7 \mathrm{~mL} \cdot \mathrm{min}^{-1} \cdot \mathrm{kg}^{-1}$; PLA: $46 \pm 5$ to $51 \pm 6 \mathrm{~mL} \cdot \mathrm{min}^{-1} \cdot \mathrm{kg}^{-1}$; time $\mathrm{x}$ treatment interaction, $\left.P=0.50\right)$. Time to complete the $10-\mathrm{km}$ time-trial was reduced by $14 \pm 7 \%$ (time effect, $P<0.001$ ), with no differences between groups (time $\mathrm{x}$ treatment interaction, $P=0.15$ ). Muscular endurance increased by $6 \pm 7 \%$ (time effect, $P<0.001$ ), with no differences between groups (time x treatment interaction, $P=0.84$ ). Whole body lean mass was unchanged over time $(P=0.097)$. However, leg lean mass showed an increase following endurance exercise training $(P<0.001)$, which tended to be greater in PRO (PRO: $0.5 \pm 0.7 \mathrm{~kg}$; PLA: $0.2 \pm 0.6 \mathrm{~kg}$; time $\mathrm{x}$ treatment interaction, $P=0.073)$. Protein supplementation after exercise and before sleep does not further augment the gains in whole-body oxidative capacity and endurance exercise performance following prolonged endurance exercise training in healthy, young males.

Low Energy Availability Assessed by a Sport-Specific Questionnaire and Clinical Interview Indicative of Bone Health, Endocrine Profile and Cycling Performance in Competitive Male Cyclists

N Keay ${ }^{1}$, G Francis ${ }^{2}$, and K Hind ${ }^{3}$

${ }^{1}$ Visiting Fellow, Department of Sport and Exercise Sciences, Durham University, UK

\author{
${ }^{2}$ Science4performance, London, UK \\ ${ }^{3}$ Department of Sport and Exercise Sciences, Durham University, UK
}

We evaluated the efficacy of a sport-specific energy availability (EA) questionnaire, combined with clinical interview, for identifying male athletes at risk of developing bone health, endocrine and performance consequences of relative energy deficiency in sports (RED-S). Fifty competitive male road cyclists, recruited through links of participants in a pilot study, were assessed by a newly developed sport-specific questionnaire and clinical interview (SEAQ-I), received dual energy X-ray absorptiometry (DXA) bone mineral density (BMD) and body composition scans and blood tests for endocrine markers. Low EA as assessed using the SEAQ-I, was observed in $28 \%$ of cyclists. Low lumbar spine BMD (Z-score $<-1.0)$ was found in $44 \%$ of cyclists. EA was the most significant determinant of lumbar spine BMD Z-score $(\mathrm{p}<0.001)$. Among low EA cyclists, lack of previous load-bearing sport was associated with the lowest BMD $(p=0.013)$. Low EA was associated with reduced total percentage fat $(\mathrm{p}<0.019)$. The 10 cyclists with chronic low EA had lower levels of testosterone compared with those having adequate EA $(\mathrm{p}=0.024)$. Mean vitamin $\mathrm{D}$ concentration was below the level recommended for athletes $(90 \mathrm{nmol} / \mathrm{l})$. Training loads were positively associated with power-to-weight ratios, assessed as 60 minute Functional Threshold Power (FTP) per kg $(\mathrm{p}<0.001)$. Percentage body fat was not significantly linked to cycling performance. This study demonstrates that a sport-specific questionnaire and clinical interview (SEAQ-I) is effective for identifying male road cyclists with acute intermittent and chronic sustained low EA. Cyclists with low EA, particularly in the long-term, displayed adverse quantifiable measures of bone, endocrinology and performance consequences of RED-S.

The Impact of a 24-h Low FODMAP Diet on Exercise-Associated Gastrointestinal Symptoms and Breath Hydrogen Responses in Endurance Athletes

SK Gaskell ${ }^{1}$, B Taylor ${ }^{1}$, J Muir ${ }^{2}$, and RJS Costa ${ }^{1}$

${ }^{1}$ Department of Nutrition Dietetics \& Food, Monash University, Australia

${ }^{2}$ Department of Gastroenterology, Monash University, Australia

Exercise-associated gastrointestinal symptoms (GIS) in endurance athletes are common and can potentially lead to debilitating exercise performances and hampering of post-exercise recovery. This study aimed to determine the impact of pre- and post-exercise dietary fermentable oligo- di- monosaccharide and polyol (FODMAP) intake on GIS and breath hydrogen $\left(\mathrm{H}_{2}\right)$ responses (marker of carbohydrate malabsorption) in response to exertional-heat stress. Using a randomised crossover and counterbalanced study design, non-heat acclimatised recreationally competitive endurance runners $(\mathrm{n}=18$; male $=10$ and female $=8)$ consumed a 24-h high $($ HFOD $)$ or low (LFOD) FODMAP diet on one occasion, prior to completing 2-h of running at $60 \% \dot{V} O_{2 \max }$ in hot ambient conditions $\left(35^{\circ} \mathrm{C}, 23 \%\right.$ relative humidity). Immediately post-exercise a recovery beverage was consumed matching the 24-h FODMAP diet. GIS and breath $\mathrm{H}_{2}$ samples were collected pre-exercise, every $15 \mathrm{~min}$ during exercise, and every $15 \mathrm{~min}$ post-exercise for 4-h. Blood samples were collected pre- and post-exercise 
to determine plasma osmolality and plasma cortisol concentrations. Participants then repeated the protocol on the alternating diet after a oneweek washout period. The results showed that GIS incidence during exertional-heat stress did not differ between LFOD and HFOD (89\%). However, compared with LFOD, the severity of GIS was significantly higher on HFOD pre- and during exercise, and in total across the day $(\mathrm{p}=.027, \mathrm{p}=.035$, and $\mathrm{p}=.042$, respectively). A significant rise in breath $\mathrm{H}_{2}$ (>10 ppm) was evident in HFOD post-exercise from 2:15-h onwards but not on LFOD. A significant difference in total breath $\mathrm{H}_{2}$ production over the 4-h period and the area under the curve was observed on HFOD (mean and 95\% CI: 2525 (1452-3597 ppm $\left.4-\mathrm{h}^{-1}\right)$, peak +17 ppm) compared to LFOD (1505 (1031-1978 $\left.\mathrm{ppm}^{4} 4 \mathrm{~h}^{-1}\right)$, peak $\left.+8 \mathrm{ppm}\right),(\mathrm{p}=.031)$. Pre- and post-exercise plasma cortisol did not differ between diets ( $p>$.05). Findings suggest that a 24-h HFOD diet prior to exertional-heat stress, and a HFOD recovery beverage immediately post-exercise, results in exacerbating exercise-associated GIS and carbohydrate malabsorption in comparison to a LFOD dietary intervention.

Individual Muscle Hypertrophy to Different Resistance Training Variations: Biological Responsivity vs Training Modulation

F Damas $^{1}$, NS Silva-Magosso ${ }^{1}$, TS Chaves ${ }^{1}, \mathrm{C}_{\text {Barcelos }^{1} \text {, OC Witard }}{ }^{2}$, and CA Libardi ${ }^{1}$

${ }^{1}$ Dept of Physical Education, Federal University of São Carlos, Brazil

${ }^{2}$ Faculty of Health Sciences and Sport, University of Stirling, Scotland

To date, it is unknown if such practice augments intra-individual RTinduced muscle hypertrophy. In this retrospective study, we aimed to examine individualised changes in vastus lateralis cross-sectional area (CSA) to distinct RT variables (ie, frequency, repetition duration and intensity) modulations over an 8-10 wk RT period. A study cohort of 34 young (18-30 y) men from 3 separate data sets each completed a single leg RT programme, ie, each participant completed two RT programmes. Data set A consisted of 10 untrained men who performed 3 sets of leg extension exercise (9-12 max reps) 5x/wk (RT5) vs 3x/wk (RT3) for 8 wk. Data set B consisted of 10 untrained men who performed 3 sets of leg extension exercise (70\% 1RM) with a fixed repetition duration (FIX, 2:2s) vs. self-selected tempo (SELF) 2x/wk for $8 \mathrm{wk}$. Data set C consisted of 14 trained men (RT experience: 5 (3)y) who performed $\sim 6$ sets of leg press +6 sets of leg extension +5 sets of leg flexion per RT session (75\% 1RM) until muscle failure (RT-F) vs. volitional interruption of each set (RT-V) $2 x / w k$ for $10 \mathrm{wk}$. Vastus lateralis CSA was measured pre- and post-RT by ultrasound imaging fitting technique and the change was compared between protocols by paired samples t-tests. Results showed similar ( $\mathrm{P}>0.05)$ increases in muscle CSA (mean (SD) in $\mathrm{cm}^{2}$ ) for: A, RT5, 2.0 (1.3) and RT3, 2.4 (1.7); B, FIX, 1.0 (0.6) and SELF, 1.3 (0.8); and C) RT-F, 4.2 (3.3) and RT-V, 4.4 (1.7). Intra-subjects analyses revealed that some subjects responded better to one of the protocols (A, RT5: 1, RT3: 5; B, FIX: 1, SELF: 5; C, RT-F: 4, RT-V: 9 subjects) but others showed no differences between legs (A, 4; B, 4; C, 1 subject). Inter-subject variability in the muscle hypertrophic response was high, with the $\mathrm{CV}$ varying from $37-77 \%$ intra-protocols. These results demonstrate that regardless of RT frequency, repetition duration, intensity manipulation or training status, the individual biological predisposition determines the muscle hypertrophic response to RT. Individually, subjects might respond better to specific RT modulations.

Effect of Carbohydrate Intake During Matches on the Cognitive Function of Professional Female Football Players

M Martínez ${ }^{1}, \mathrm{~N}$ Bonfanti $^{2}$, and S Galloway ${ }^{3}$

${ }^{1}$ Universidad Europea de Madrid, Spain
${ }^{2}$ Universidad San Jorge, Saragossa, Spain

${ }^{3}$ University of Stirling, Scotland, UK

Football involves an intermittent high-intensity movement pattern over a prolonged period of time. Carbohydrates $(\mathrm{CHO})$ are the main fuel for intense training sessions and matches. Previous studies have shown a reduction of fatigue alongside a retention of cognitive performance in football players ingesting $\mathrm{CHO}$ during trials conducted in laboratory settings. However, little work has examined female players or whether taking $\mathrm{CHO}$ during matches can have similar effects in an applied setting. The current study aimed to determine if a $12 \% \mathrm{CHO}$ beverage taken before and at half time in a football match could aid retention of cognitive function and reduce fatigue, in female football players. 6 Spanish players played 2 football matches on separate days. The players ingested $250 \mathrm{ml}$ during the warm-up and $250 \mathrm{ml}$ at halftime of either a non-caloric placebo beverage (PLA) or a 12\% maltodextrin beverage $(\mathrm{CHO})$. Both beverages were matched for taste, odor and color. Gastric distress and gut fullness (GD, GF), session rate of perceived exertion (S-RPE;), fatigue () and mood (MS) were recorded before the match, during half-time, and at the end of the match. Urine color (UC) and body mass were assessed before the warm up and at the end of the match. Ball possession (BP) and sprints were recorded during matches. Players completed the Stroop Color cognitive function test and a mental concentration test at the end of the match. All data were analyzed by Wilcoxon signed-rank test. S-RPE was higher in CHO than in PLA at half-time $(p=0.03)$ and at the end of the match $(p=0.03)$. Fatigue increased between the half-time and the end of the match when players took PLA $(p=0.04)$. Mass loss over the match was significantly higher in $\mathrm{CHO}$ trial $(\mathrm{p}=0.04)$. No differences were found for GD, GF, MS, BP, S, or cognitive function and mental concentration. In conclusion, taking $250 \mathrm{ml}$ of a $12 \% \mathrm{CHO}$ beverage during warm up and at half-time of football matches with similar game analysis aspects did not impact upon gastrointestinal symptoms, cognitive function, or mental concentration in professional female soccer players, which could be related with the different S-RPE of each match that cannot be controlled during an ecological valid setting.

Estimated Energy Balance of International Female Rugby Sevens Players in Training and Competition Scenarios

$\mathrm{C}_{\text {Curtis }}{ }^{1,2}, \mathrm{~N}$ Arjomandkhah ${ }^{1}, \mathrm{C}$ Cooke $^{1}$, and M Russell ${ }^{1}$

${ }^{1}$ School of Social and Health Sciences, Leeds Trinity University, UK

${ }^{2}$ London Sports Institute, Middlesex University, UK

Rugby sevens is a contact sport comprising of two teams of seven players who compete over two 7-min halves. Rugby sevens players experience accentuated loads during match-play, with mean total distances of $\sim 113$ $120 \mathrm{~m} \cdot \mathrm{min}^{-1}$ covered. Likewise, $\sim 30 \%$ of total match distances are covered at speeds $\geq 5 \mathrm{~m} \cdot \mathrm{s}^{-1}$, while $39 \%$ more high velocity $\left(\geq 4 \mathrm{~m} \cdot \mathrm{s}^{-2}\right)$ accelerations are performed when players compete in international tournaments. Accordingly, to facilitate optimised performance during match-play and realisation of training-induced adaptations, the energy balance of rugby sevens players needs consideration. However, limited data currently exists regarding the energy intake (EI) and expenditure (EE) of rugby sevens players in both training and competitive scenarios, especially in the case of female players. Therefore, this two-part study aimed to estimate EI and EE in international female rugby sevens players during a) a training camp $(n=11)$, and $\mathrm{b})$ an international competition $(n=8)$ held within 14 days of each other. Tri-axial accelerometers (Actigraph wGT3X-BT, USA) and 5-day food diaries (quantifying portion sizes using household measures) facilitated estimation of $\mathrm{EE}$ and EI, respectively. Activity-based EE was combined with body mass-derived estimates of resting EE to estimate total daily $\mathrm{EE}$ (TDEE). Energy deficits of $-47 \%$ (TDEE $_{\text {Training: }}$ : $\left.3502 \pm 400 \mathrm{kcal} \cdot \mathrm{d}^{-1}, \mathrm{EI}_{\text {Training: }}: 1859 \pm 221 \mathrm{kcal} \cdot \mathrm{d}^{-1}, \mathrm{p} \leq 0.001, \mathrm{~d}=5.1\right)$ and 


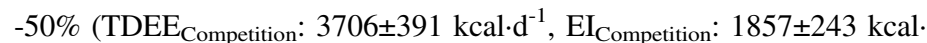
$\left.\mathrm{d}^{-1}, \mathrm{p} \leq 0.001, \mathrm{~d}=5.7\right)$ were observed throughout training and competition. Similarly, CHO intakes were lower than sports nutrition recommendations in training $\left(-62 \% ; 2.3 \pm 0.3 \mathrm{~g} \cdot \mathrm{kg}^{-1} \cdot \mathrm{BM}^{-1}, \mathrm{p} \leq 0.001\right)$ and competitive $\left(-60 \% ; 2.4 \pm 0.5 \mathrm{~g} \cdot \mathrm{kg}^{-1} \cdot \mathrm{BM}^{-1}, \mathrm{p} \leq 0.001\right)$ scenarios whereas for protein $\left(1.7 \pm 0.4 \mathrm{~g} \cdot \mathrm{kg}^{-1} \cdot \mathrm{BM}^{-1}, 1.5 \pm 0.1 \mathrm{~g} \cdot \mathrm{kg}^{-1} \cdot \mathrm{BM}^{-1}\right)$ and fat $(35 \pm 5 \%, 39 \pm 6 \%$ total energy) intakes exceeded the lower range of recommendations in both training and competition, respectively. In international female rugby sevens players, these findings indicate that estimated EE exceeds EI in both competition and training and that carbohydrate and fat intakes are less than optimal when compared to current performance-based sports nutrition recommendations.

\section{Day 2-Orals: Health Theme}

\section{Prevalence of Risk Factors for Eating Disorders Among Semi-Professional Team Sports Players}

D Baldó $^{1}, \mathrm{~N}$ Bonfanti $^{2}$

${ }^{1}$ General Services, Health and Sports, Banco Santander, Madrid, Spain

${ }^{2}$ Health Sciences Faculty, San Jorge University, Zaragoza, Spain

Eating Disorders (EDs) are characterized by an extreme concern about body weight and body image which can lead to serious disease and even death. Male team sports players are usually excluded from the population at risk of developing EDs. This exemption can be questioned based on previous studies identifying a great body concern in male team athletes along with a generalized increase of extreme concern about body image and the sport-thinness relation. The current study aimed to detect EDs symptoms and risk factors for its development in male team sports players. A descriptiveanalytical study was carried out with 49 male adult semi-professional team sports players (age $25.6 \pm 4.5 \mathrm{y}$, body weight $80.8 \pm 11.4 \mathrm{Kg}$, height $1.8 \pm 7.7 \mathrm{~m}$, BMI $24.2 \pm 2.5$ ). Data was collected through a general assessment sheet along with the Eating Habits Questionnaire for Athletes to assess the concern for body weight, body shape and food intake by factors related to sports (CHAD), the Test of Food Attitudes to detect the symptoms of anorexia nervosa (EAT-40), the Inventory of Eating Disorders to contemplate cognitive-behavioral characteristics of EDs (EDI-2), and the Questionnaire on the Body Shape to evaluate attitudinal alterations about the body image (BSQ; Avila et al., 1996). Subjects were grouped based on their age, BMI, sports modality, playing position, training load, sports casualties and perceived pressure level. All data were analyzed with a Kolmogorov-Smirnov, Student's t, ANOVA and Pearson correlation. It was observed that $14 \%$ of the subjects presented symptoms of EDs. Positive correlation was observed between BMI and the CHAD score $(\mathrm{p}=0.04)$ and between BMI and the BSQ score $(p=0.02)$. CHAD $(p=0.00)$, EAT-40 $(p=0.03)$, EDI-2 $(p=0.01)$ and BSQ $(p=0.03)$ were correlated positively with the weekly training load. Soccer players and rugby players were found to score significantly higher than basketball players on the EAT- $40(\mathrm{p}=0.04, \mathrm{p}=0.07$ respectively) and CHAD ( $p=0.12, p=0.08$, respectively). In conclusion, male sports team players could also be a group of risk for EDs development, being soccer and rugby the sport with greater risk. In addition, it seems that the risk for EDs is greater when BMI or training load are greater.

Validation of the ActiGraph GT3X-BT Accelerometer for Step Counts at Five Different Body Locations in Laboratory Settings

A Karaca ${ }^{1}, \mathrm{~N}$ Demirci $^{1}$, V Yilmaz ${ }^{2}$, S Hazır Aytar ${ }^{3}, \mathrm{~S} \mathrm{Can}{ }^{4}$, and E Ünver ${ }^{5}$

${ }^{1}$ Division of Physical Activity and Health, Department of Recreation, Faculty of Sport Sciences, Hacettepe University, Turkey
${ }^{2}$ Division of Sports Biomechanics and Motor Control, Department of Exercise and Sport Sciences, Faculty of Sport Sciences, Hacettepe University, Turkey

${ }^{3}$ Department of Sport Sciences, Faculty of Health Sciences, Başkent University, Turkey

${ }^{4}$ Department of Sport Sciences, Faculty of Sport Sciences, Hitit University, Turkey

${ }^{5}$ Division of Sports and Coaching, Department of Exercise and Sport Sciences, Faculty of Sport Sciences, Hacettepe University, Turkey

The aim of this study was to determine the validation of the ActiGraph GT3X-BT accelerometer for step counts at five different body locations in laboratory settings. Digital image processing code was written in MathLab. The code is calculating with $100 \%$ accuracy of the actual step counts for each speed during an incremental walking and running bout on a treadmill. A total of 29 male (age:26.2 \pm 6.2 yrs) participated in this study. Participants were asked to wear the ActiGraph GT3X-BT accelerometers on their left and right wrists, waist, right ankle, and right upper arm. Participants walked or run for two minutes for each speed $(2,4,6,8,10 \mathrm{~km} / \mathrm{h}$; total 10 minutes) on the treadmill while wearing five ActiGraph GT3X-BT accelerometers on their body. Step counts (SC) were recorded simultaneously by using both video camera for calculating digital image processing code and each ActiGraph GT3X-BT accelerometer. There was a significant correlation between Actual SC and Ankle SC during $4 \mathrm{~km} / \mathrm{h}$ (ICC and 95\% CI: 0.964 (0.923-0.983). There were significant correlations between Actual SC and Waist SC for different speeds (ICC and 95\% CI: 6 km/h: 0.986 (0.971-0.994); 8 km/h: 0.995 (0.990-0.998); 10 km/h: 0.966 (0.928-0.984)). ICC and 95\% CI between the Actual SC and the Upper Arm SC were 0.964 (0.923-0.983) and 0.991 (0.980-0.996), respectively, at 6 and $8 \mathrm{~km} / \mathrm{h}$. There were significant correlations between the average of Actual SC and the average of Waist SC (ICC and 95\% CI: 0.913 (0.816$0.959)$, and also between the average of Actual SC and the average of Upper Arm SC (ICC and 95\% CI: 0.839 (0.657-0.924). Similarly, there were excellent agreements with Bland-Altman plots the waist $(6,8$ and $10 \mathrm{~km} / \mathrm{h})$, the ankle $(4 \mathrm{~km} / \mathrm{h})$ and the upper arm $(6$ and $8 \mathrm{~km} / \mathrm{h})$. In conclusion; the waist and the upper arm provide the best assessment of the average step count during walking and running in the laboratory setting.

Effects of Daily and Intermittent Intake of New Zealand Blackcurrant Extract on Whole-Body Fat Oxidation During Moderate Intensity Exercise in Males

MA Şahin ${ }^{1,2}$, P Bilgiç $^{2}, \mathrm{~S}$ Montanari ${ }^{1}$, and MET Willems ${ }^{1}$

${ }^{1}$ Institute of Sport, University of Chichester, Chichester, UK

${ }^{2}$ Department of Nutrition and Dietetics, Hacettepe University, Ankara, Turkey

Seven days intake of anthocyanin-rich New Zealand blackcurrant (NZBC) extract increased exercise performance and enhanced exercise-induced fat oxidation. Elimination of anthocyanin metabolites can take several days. It is not known whether daily intake of NZBC extract is required for effectiveness. We examined the effect of daily and intermittent NZBC extract intake on metabolic and physiological responses during brisk walking. Sixteen physically active healthy males (age: $24 \pm 6$ y, body mass: $78 \pm 16 \mathrm{~kg}$, BMI: $24.7 \pm 4.2 \mathrm{~kg} \cdot \mathrm{m}^{-2}$, IPAQ score: $\left.4385 \pm 1635 \mathrm{Met} \cdot \mathrm{week}^{-1}\right)$ volunteered. Resting metabolic equivalent (1-MET) was measured by using Douglas bags (1-MET: $\left.3.95 \pm 0.64 \mathrm{ml} \cdot \mathrm{kg}^{-1} \cdot \mathrm{min}^{-1}\right)$. Then, an incremental walking test was performed to calculate the relationship between walking speed and MET. A randomised, cross-over experimental design was used for baseline, and 14-day intermittent (14I) and 14-day continuous (14C) intake. Participants consumed 2 capsules of NZBC extract $(600 \mathrm{mg}$ 
and containing $210 \mathrm{mg}$ of anthocyanins, (CurraNZ ${ }^{\mathrm{TM}}$ Health Currancy Ltd., Surrey, UK) with breakfast daily for 14 days or every other day for 14 days with a 14-day washout. On the morning of the final intake, participants ingested two capsules 2 -hr before the 30 -min brisk walk at $4(\mathrm{n}=3)$ or $5(\mathrm{n}=13)$ METs (speed: $\left.5.66 \pm 0.65 \mathrm{~km} \cdot \mathrm{hr}^{-1}\right)$. Responses were measured at 7-10, 17-20 and 27-30 min during the walk and averaged and analysed with one-way repeated measures ANOVA and post-hoc student t-tests. There were no changes in heart rate (baseline: 102 $\pm 17,14 \mathrm{I}: 101 \pm 18$, 14C: $101 \pm 18$ beats $\cdot \mathrm{min}^{-1}$ ) and oxygen uptake (STPD) (baseline: $1.50 \pm 0.3$, 14I: $\left.1.52 \pm 0.32,14 \mathrm{C}: 1.51 \pm 0.33 \mathrm{~L} \cdot \mathrm{min}^{-1}\right)$. Daily intake showed a trend for lower RER (baseline: $0.85 \pm 0.05,14 \mathrm{I}$ : $0.85 \pm 0.04,14 \mathrm{C}$ : $0.84 \pm 0.04$, $\mathrm{P}=0.07$ ) and lower carbohydrate oxidation (baseline: $0.95 \pm 0.40,14 \mathrm{I}$ : $\left.0.95 \pm 0.34,14 \mathrm{C}: 0.86 \pm 0.33 \mathrm{~g} \cdot \mathrm{min}^{-1}, \mathrm{P}=0.07\right)$. Enhanced fat oxidation by $16 \%$ was observed only for 14-day daily intake (baseline: $0.36 \pm 0.12$, 14I: $\left.0.37 \pm 0.10,14 \mathrm{C}: 0.41 \pm 0.13 \mathrm{~g} \cdot \mathrm{min}^{-1}, \mathrm{P}=0.04\right)$. Daily intake of New Zealand blackcurrant extract is required to enhance fat oxidation during brisk walking instead of intake every other day. Our findings seem to indicate that a build-up and maintenance of anthocyanin metabolites is required for effectiveness of New Zealand blackcurrant extract.

Acknowledgement: We thank Health Currancy (UK) Ltd and CurraNZ (NZ) Ltd for supplements and Blackcurrant New Zealand Inc. for support for conference attendance.

The Effects of High Intensity Interval Exercise on $\mathrm{VO}_{2 \max }$, Endurance Capacity and Fat Oxidation; "Double" HIIT versus "Single" HIIT MM Atakan ${ }^{1}$, Y Güzel ${ }^{1}$, S Bulut ${ }^{1}$, ȘN Koşar ${ }^{1}$, and HH Turnagöl ${ }^{1}$

${ }^{1}$ Nutrition and Metabolism in Exercise Division, Faculty of Sport Sciences, Hacettepe University, Turkey

High intensity interval training (HIIT) results in similar training adaptations associated with continuous aerobic training. Additionally, HIIT is an effective strategy to affect a number of health outcomes including increases in cardiorespiratory fitness and maximal fat oxidation, upregulation of skeletal muscle proteins and markers of mitochondrial function related to oxidative phosphorylation. Further to this, the shortest HIIT studies involving a total of 6 HIIT sessions over 2 weeks improved $\mathrm{VO}_{2 \max }$, endurance performance and fat oxidation. However, the effects of such a HIIT program undertaken twice daily for one week on these parameters are unclear. Thus, the purpose of this study is to compare the effects of HIIT programs over 2 weeks with a HIIT protocol consisting of total of 6 sessions performed on daily twice every second day on $\mathrm{VO}_{2 \max }$, endurance performance and fat oxidation: The first involving a single session per day for two weeks, the second involving double sessions per day for one week. Twenty-eight active males (age:25,17 $\pm 4,3 \mathrm{yrs}$ ) were assigned to either the double HIIT (HIIT-D, $n=15$ ) or the single HIIT (HIIT-S, $n=13$ ) groups. HIIT-D performed two consecutive training sessions on the same day, separated by 3 h. HIIT-S was trained once per day, 3 days/week for 2 wk. Both groups completed 6 HIIT sessions. Each training session consisted of $10 * 60$ s repeated efforts of cycling at $\mathrm{VO}_{2 \max }$, interspersed by $75 \mathrm{~s}$ of cycling at low intensity $(30 \% \mathrm{VO} 2 \mathrm{max})$. Before and after the training program, $\mathrm{VO}_{2 \max }$, endurance performance and fat oxidation during exercise were measured. All tests and training protocols were conducted on a cycle ergometer and VO2 was measured by an oxygen analyzer. Repeated measure ANOVA was used to analyze the effects of different HIIT intervention and group differences. $\mathrm{VO}_{2 \max }$ (HIIT-D 8\%, HIIT-S 6\%) and endurance performance (HIIT-D 76\%, HIIT-S 59\%) were improved $(\mathrm{p}<0.05)$ in both groups, but the magnitude of the improvements in $\mathrm{VO}_{2 \max }$ and endurance capacity were similar $(\mathrm{p}>0,05)$. Increase in fat oxidation was significant in HIIT-D $(13 \%$; $\mathrm{p}<0.05)$, while not significant in HIIT-S $(9 \%$; $>0.05)$. The short-term HIIT program undertaken twice a day for only 1 week is as effective as the 2 -week program to improve $\mathrm{VO}_{2 \max }$ and endurance capacity. However, the effect of the double HIIT on fat oxidation is greater.

\section{Day 2-Case Studies - Oral Presentations}

Making Weight Safely: Assessment of Within Daily Energy Balance and Manipulation of Energy Availability Without Symptoms of RED-S in an Elite Male Taekwondo Athlete

C Langan-Evans ${ }^{1}$, M Germaine ${ }^{1}$, M Artukovic ${ }^{1}$, SO Shepherd ${ }^{1}$, GL Close $^{1}$, and JP Morton ${ }^{1}$

${ }^{1}$ Research Institute for Sport and Exercise Sciences, Liverpool John Moores University, UK

Combat sport athletes involved in weight making may experience transient periods of low energy availability (LEA), resulting in symptoms of the relative energy deficiency in sport syndrome (RED-S), whilst additionally practising acute weight loss (AWL) methods, negatively affecting health and performance. The aim of this case study was to outline the impact of making weight (using novel within daily energy balance assessment) on symptoms of RED-S in a male international level Taekwondo (TKD) athlete. Over an 8 week period, the athlete adhered to a daily energy intake (EI) (1,690 kcal.day-1; 2.3g.kg-1 PRO, 3.4g.kg-1 CHO and 0.9g.kg-1 FAT) equating to resting metabolic rate (RMR). Body composition via Dual X-ray Absorptiometry, RMR and VO2peak, (utilising indirect calorimetry), venous blood sampling, cardiac scanning, strength (1-RM bench press/back squat), power (force velocity profile) and total mood disturbance (TMD) via a profile of mood states (POMS) assessments were made at regular intervals. Training consisted of specific TKD and general training sessions equating to 12-15 h.wk-1. Exercise energy expenditure (EEE) was assessed using portable actigraphy. EA ranged from $-7 \pm 1$ to $30 \pm 1 \mathrm{kcal} . \mathrm{kg}$.LBM-1.day-1. Average body mass (BM) loss was $1.0 \pm 0.8 \mathrm{~kg} . w \mathrm{k}-1$, equating to a total loss of $9.8 \mathrm{~kg}$, resulting in a decrease of fat mass $(11.4 \mathrm{~kg}-6.5 \mathrm{~kg})$. Lean body mass remained constant $(54.5-$ $55.0 \mathrm{~kg})$ and RMR was generally stable $(1710 \pm 77-1602 \pm 75 \mathrm{kcal}$.day -1$)$ eliciting minimal adaptive thermogenesis. The final $1.8 \mathrm{~kg}$ of BM loss (2.8\%) was achieved 24 hours prior to weigh in utilising active/passive dehydration methods. Despite LEA, measures of cardiovascular (VO2peak 3.9L.min-1-4.4L.min-1), strength (Bench 1.1-1.3/Squat 1.6-1.9 x BM) and increases in $\mathrm{F} / \mathrm{V}$ power performance all improved with no detrimental effect on various cardiac, endocrine, bone turnover biomarkers or psychological (TMD <2-28) health status. This strategy represented a major change in the athlete's habitual practices and demonstrates that a gradual approach to making weight can be successfully achieved via a combination of restricted and periodised EI/EEE and limited AWL techniques, whilst avoiding the negative consequences associated with LEA and RED-S. However, consideration must be given to post-competition rebound hyperphagia.

\section{Day 1-Posters}

Effects of New Zealand Blackcurrant Extract Intake on Metabolic and Physiological Responses During Moderate Intensity Exercise in Caucasian and Thai Males

MET Willems ${ }^{1}$, MA Sahin ${ }^{1,2}, \mathrm{~N}$ Parktin $^{3}$, W Widjaja ${ }^{3}$, and A Ajjimaporn $^{3}$

${ }^{1}$ Institute of Sport, University of Chichester, Chichester, UK

${ }^{2}$ Department of Nutrition and Dietetics, Hacettepe University, Ankara, Turkey 
${ }^{3}$ College of Sports Science and Technology, Mahidol University, Nakhon Pathom, Thailand

Enhanced exercise-induced fat oxidation was shown with intake of New Zealand blackcurrant (NZBC) extract in Caucasian males and females. Ethnicity may affect responses to supplementation. We examined effects of NZBC extract on metabolic and physiological responses during moderate intensity exercise in Caucasian and Southeast Asian males. Seventeen healthy Thai (T) (age $22 \pm 3$ years, BMI: $21.8 \pm 1.1 \mathrm{~kg} \cdot \mathrm{m}^{-2}$ ) and sixteen healthy Caucasian (C) males $\left(24 \pm 6 \mathrm{y}\right.$, BMI: $\left.24.7 \pm 4.2 \mathrm{~kg} \cdot \mathrm{m}^{-2}\right)$ volunteered. Data for Thai and Caucasian males were obtained at Mahidol University (Thailand) and the University of Chichester (United Kingdom), respectively. In the 1st visit, metabolic equivalent at rest (1-MET) was measured and an incremental walking protocol was completed to establish the relationship between walking speed and MET. In the 2nd and 3rd visit (randomized cross-over design), metabolic and physiological responses were measured during a 30-min treadmill walk at 5-MET except for three Caucasian males at 4-MET (speed, T: $6.1 \pm 0.5$, C: $5.7 \pm 0.7 \mathrm{~km} \cdot \mathrm{hr}^{-1}$ ). Supplementation consisted of 7-day intake of two capsules of NZBC extract (600 mg CurraNZ, Health Currancy Ltd, UK; capsules contain 35\% blackcurrant extract powder). One-MET for Thai and Caucasian males (T: $\left.4.04 \pm 0.44, \mathrm{C}: 3.95 \pm 0.64 \mathrm{ml} \cdot \mathrm{kg}^{-1} \cdot \mathrm{min}^{-1}\right)$ were similar $(\mathrm{P}=0.58)$. Heart rate during brisk walking was higher in Thai males (T: $113 \pm 14$; $\left.\mathrm{C}: 102 \pm 17 \mathrm{~b} \cdot \mathrm{min}^{-1}, \mathrm{P}=0.047\right)$. In the control condition, there was a trend for carbohydrate oxidation during brisk walking to be higher in Thai males (T: $1.21 \pm 0.37 ; \mathrm{C}: 0.95 \pm 0.40 \mathrm{~g} \cdot \mathrm{min}^{-1}, \mathrm{P}=0.06$ ). NZBC extract had no effect on carbohydrate oxidation in both groups. During brisk walking, Thai males had higher RER in the control condition (T: 0.92 $\pm 0.06 ; \mathrm{C}: 0.85$ $\pm 0.04), \mathrm{P}<0.01)$. NZBC had no effect on the RER during brisk walking in both groups. In the control condition, fat oxidation during brisk walking was lower for Thai males (T: $0.18 \pm 0.13, \mathrm{C}: 0.36 \pm 0.12 \mathrm{~g} \cdot \mathrm{min}^{-1}, \mathrm{P}<0.001$ ). NZBC extract increased fat oxidation during brisk walking only in Caucasian males by $10 \%$ (T: $0.18 \pm 0.10, \mathrm{C}: 0.39 \pm 0.13 \mathrm{~g} \cdot \mathrm{min}^{-1}, \mathrm{P}<0.05$ ). Our finding of enhanced exercise-induced fat oxidation by intake of New Zealand blackcurrant extract in Caucasian but not in Thai males suggest that the metabolic responses to anthocyanin intake may be ethnicity-dependent.

Acknowledgement: We thank Health Currancy (UK) Ltd and CurraNZ (NZ) Ltd for supplements and Blackcurrant New Zealand Inc. for support for conference attendance.

\section{Prevalence, Predictors and Extent of Dietary and Nutritional Supplement Use in the Australian Army}

B Baker ${ }^{1}$, B Probert ${ }^{1}$, D Pomeroy ${ }^{2}$, J Carins ${ }^{1,3}$, and K Tooley ${ }^{2}$

${ }^{1}$ Food and Nutrition, Land Division, Defence Science and Technology Group, Australia

${ }^{2}$ Cognition and Behaviour, Land Division, Defence Science and Technology Group, Australia

${ }^{3}$ Social Marketing, Griffith University, Australia

Dietary supplements (DSs) are designed to complement the diet with vitamins, minerals, protein or amino acids, oils, and other substances (plant, animal or synthetic). Nutritional supplements (NSs), which include sports drinks, energy bars and gels, and meal-replacement beverages, are designed for use before, during and after exercise. This study aimed to understand DS and NS use by active-duty Australian soldiers using a voluntary, anonymous cross-sectional survey, which included 70 generic products, 'other supplement' spaces, and a scale to indicate frequency of use (never, once a month, once a week, 2-6 times per week or daily).
An online survey link was e-mailed to all soldiers aged $\geq 18$ years $(n=22,743)$. Hard copies were also distributed to potential participants at 6 regiments $(n=452)$. A total of 2,162 completed responses were collected (1833 males and 296 females), comprising 9.5\% of the total population. Overall, $76.4 \%$ of male and $86.8 \%$ of female soldiers regularly used DSs (i.e. at least weekly). Logistic regression revealed the number of strength training sessions/week significantly predicted more prevalent regular use of multi-vitamins and multi-minerals $(\mathrm{p}<.001)$, individual vitamins and minerals $(p<.001)$, protein or amino acids $(p<.001)$, purported prohormones $(p<.001)$, herbal supplements $(p<.01)$, combination products $(\mathrm{p}<.001)$, and other DSs $(\mathrm{p}<.001)$. Negative binomial regression found hours/week of cardiorespiratory exercise was a significant predictor of the number of DSs used $(p<.001)$; overall $10.5 \%$ of respondents undertook 7.5 (SD 7.9) hours of cardiorespiratory exercise/ week and used $\geq 10$ products simultaneously. BMI range was a significant predictor of the use of combination products $(\mathrm{p}<.05)$ and joint health products $(\mathrm{p}<.01)$. Military characteristics which significantly predicted the number of DSs used were rank and engagement in special operations; almost $15 \%$ of some subgroups within these variables used $\geq 10$ products simultaneously. Rank was also a significant predictor of the number of NSs respondents used. The results suggest strategies (e.g. social marketing) to improve the quality of supplement use should be investigated. Effective strategies would motivate a reduction in unsafe supplement use and promote the use of independently tested, safe and effective supplements.

\section{Measured Versus Predicted Resting Metabolic Rate (RMR) in Elite Rugby 7s Players}

KE Black ${ }^{1}$, S Sims ${ }^{2}$, D Baker ${ }^{1}$, K Schofield ${ }^{2}$, J McQuillan ${ }^{2}$, H Thorpe ${ }^{2}$

${ }^{1}$ Department of Human Nutrition, University of Otago, PO Box 56, Dunedin, New Zealand

${ }^{2}$ Te Oranga School of Human Development and Movement Studies, University of Waikato, Hamilton, New Zealand

To estimate the energy needs of an individual an indication of their energy expenditure is required which includes Resting Metabolic Rate (RMR). However, measuring RMR in the field is difficult due to the equipment and strict protocols that need to be adhered to. Therefore, predictive equations have been created to estimate RMR as a baseline measurement for energy needs have been generated. However, the accuracy of these measures amongst elite female rugby players has not yet been assessed. The aim of the present study was to determine the accuracy of commonly used RMR prediction equations compared to the direct measurement of RMR amongst elite female rugby $7 \mathrm{~s}$ players. Twenty one elite female rugby $7 \mathrm{~s}$ players provided written informed consent to participate in the study. Participants drove to the clinic following an overnight fast, where RMR, height, weight and a DEXA scan measures were taken. Resting metabolic rate was predicted $(\mathrm{kcal} / 24 \mathrm{~h})$ from a variety of equations; Haris-Benedict, $=655.96+1.850 *$ height $(\mathrm{cm})+9.563 *$ weight $(\mathrm{kg})-4.676 *$ age $(\mathrm{y})$; Miffin St Jeor, = $10 \mathrm{x}$ weight $(\mathrm{kg})+6.25 \mathrm{x}$ height $(\mathrm{cm})-5 \mathrm{x}$ age $(\mathrm{y})-161$; Owen, = $(795+7.18 *$ weight $(\mathrm{kg})) ;$ Schofield,$=(34 *$ weight $(\mathrm{kg})+0.06 *$ height $(\mathrm{cm})+$ $3530) / 4.184$ and Cunningham, $=500+22 *$ FFM $(\mathrm{kg})$. A one-way repeated measures analysis of variance (ANOVA) was used to compare differences among measured RMR and predicted RMR values. Follow up analysis included paired sample t-tests with Bonferroni adjustment $(0.05 / 5=0.01)$. Data presented as mean \pm standard deviation. Only the Cunningham equation predicted RMR $(1704 \pm 129 \mathrm{kcal} / 24 \mathrm{~h})$ that was not significantly different from measured RMR, $1744 \pm 206 \mathrm{kcal} / 24 \mathrm{~h}$ ( $\mathrm{p}>0.999$ ), with a mean difference of $39 \mathrm{kcal}$. The Schofield equation $(1588 \pm 121 \mathrm{kcal} / 24 \mathrm{~h})$ although statistically different to the measure RMR $(p=0.008)$ the mean difference was small $(156 \mathrm{kcal})$ suggesting a possible alternative to the Cunningham 
equation accurate measures of body composition are unavailable. As previously reported in the literature all the prediction equations significantly underestimated RMR compared to measured values (range 39-401 kcal). In line with other athletic populations the Cunningham equation appears to be the most accurate for predicting resting metabolic rate in female $7 \mathrm{~s}$ players.

\section{Reliability of Three Urine Specific Gravity Meters Measuring Urine at Different Temperatures}

F Wardenaar ${ }^{1}$, C P Ortega-Santos ${ }^{1}, \mathrm{~K}$ Vento $^{1}$, S Olzinski ${ }^{1}$, C Johnston ${ }^{1}$, and $\mathrm{S}$ Kavouras ${ }^{1}$

${ }^{1}$ College of Health Solutions, Arizona State University, USA

In athletic performance, a reliable tool used on the field to assess hydration status is the urine refractometer. Urine density is influenced by the temperature and preferably measured at a standard temperature of $20^{\circ} \mathrm{C}$. Manufactures of refractometers state that reliable measurements can be performed in solutions with temperatures between $5-37^{\circ} \mathrm{C}$. The current study aimed to evaluate the outcome of three urine refractometers at different temperatures.In this study we used a manual hydrometer (HYD, Fishers Sci, USA), a digital PEN-S.G. (DIG, Atago Co, Ltd, Tokyo, Japan) and a manual refractometer (MAN, RHC-200ATC, WMicro, USA) according to the manufacturers' instructions. Healthy adults delivered 33 pre- and post-exercise urine samples that were stored at $5^{\circ} \mathrm{C}$ and measured within 4 days at $5^{\circ} \mathrm{C}, 20^{\circ} \mathrm{C}$ and $37^{\circ} \mathrm{C}$ using $30 \mathrm{~mL}$ of urine. No mean differences $(\mathrm{P}>0.05)$ were seen for DIG at $5^{\circ} \mathrm{C}(0.0000$ [95\%CI: $-0.0001,0.0002])$ and $\mathrm{MAN}$ at $20^{\circ} \mathrm{C}$ and $5^{\circ} \mathrm{C}(-0.0001$ [95\% CI: $-0.0004,0.0002$ ] and -0.0002 [95\%CI: -0.0009.0.0004] respectively), but HYD measurements at all temperatures were different from DIG $20^{\circ} \mathrm{C}$ $(\mathrm{P}<0.001)$ showing the smallest mean difference at $20^{\circ} \mathrm{C}(0.0022$ [95\% CI: $0.0012,0.0033]$. The outcome of all refractometers differed significantly at $37^{\circ} \mathrm{C}$ from other temperatures $(\mathrm{P}<0.001)$ resulting in slightly over reporting of MAN (-.0005 [95\%CI: $-0.0008,-0.0002)$ and under reporting of HYD $(0.0058$ [95\%CI: $0.0051,0.0066)$ at $37^{\circ} \mathrm{C}(\mathrm{P}<0.001)$. All methods showed a high correlation at all temperatures with each other (range: $\mathrm{r}=0.953-1.000$ ). Bland-Altman plots showed that HYD data underreported hydration status consistently at $5^{\circ} \mathrm{C}$ and $20^{\circ} \mathrm{C}$. At $37^{\circ} \mathrm{C}$ all tools showed higher urine specific gravity (USG)-values resulting in a larger magnitude of misreporting. Contingency tables showed that HYD outcomes misreported hydration status in comparison to DIG at $20^{\circ} \mathrm{C}$ with increasing temperature indicating that $9 \%\left(5^{\circ} \mathrm{C}\right), 12 \%\left(20^{\circ} \mathrm{C}\right)$ and $24 \%$ $\left(37^{\circ} \mathrm{C}\right)$ were falsely negative, being diagnosed as euhydrated instead of dehydrated. In conclusion, USG measurements at $37^{\circ} \mathrm{C}$ are not reliable to assess hydration status. The hydrometer does classify a substantial number of samples being euhydrated in comparison with DIG and MAN. Results suggest that DIG and MAN measurements at $5^{\circ} \mathrm{C}$ and $20^{\circ} \mathrm{C}$ provide comparable outcomes.

Including Environmental Impact Principles into Sports Nutrition. Case Study: the Athlete's Plate in Switzerland

A Reguant-Closa ${ }^{1}, \mathrm{~J}_{\text {Lansche }}{ }^{2}$, T Nemecek ${ }^{2}$, A Roesch $^{2}$, and NL Meyer ${ }^{3}$

${ }^{1}$ University of Andorra, International $\mathrm{PhD}$ program, Principality of Andorra

${ }^{2}$ Agroscope, LCA research group, Zurich, Switzerland

${ }^{3}$ Beth-El College of Nursing and Health Sciences, Department of Health Sciences, University of Colorado, Colorado Springs, USA

Periodized nutrition is necessary to optimize training intensities and volumes through the season. The Athlete's Plate (AP) is a nutrition education tool developed to educate athletes on how to design their plates depending on training loads (Easy (E), Moderate (M) and Hard (H)).
The AP was validated showing significantly higher protein content than recommended (up to $2.9+0.5 \mathrm{~g} \cdot \mathrm{kg}^{-1}$ BodyMass $\cdot$ day $^{-1} ; \mathrm{p}<0.001$ ). The aim of this study was to evaluate the protein content and the environmental impact (EI) of the AP in Switzerland and give recommendations to decrease it, while maintaining an adequate diet for sports performance and health. Nutritional content of 216 AP created by 12 Registered Sports Dietitians were evaluated using Computrition Software (Hospitality Suite, v. 18.1, Chatsworth, California). The EI of the plates was analyzed by Life Cycle Assessment (LCA), according to SALCA methodology. Results showed a higher animal protein content on the plates $(70 \%)$, mainly coming from animal and dairy, vs vegetarian sources (30\%). A higher EI is directly linked with higher training intensities $\mathrm{E}(5.7+2.9 \mathrm{kgCO} 2 \mathrm{eq} / \mathrm{day}) ; \mathrm{M}(6.4+1.6 \mathrm{kgCO} 2 \mathrm{eq} / \mathrm{day} ;)$ and $\mathrm{H}(8.0+2.1 \mathrm{kgCO} 2 \mathrm{eq} /$ day $)$. However, there was a big variability on the EI of the plates depending on the composition and amounts of food. This study will permit to include recommendations on how to create an AP with low EI while maintaining and adequate nutrition for performance and health. With a raising interest on the link between environmental impact of food, health and performance, also among professional athletes, this adapted tool is a good framework for education and improvement on this direction.

\section{Are Predictive Equations Valid for Resting Metabolic Rate Estimation in Former Elite Athletes Using Indirect Calorimetry as the Reference Method?}

F Jesus $^{1,2}$, C Nunes ${ }^{1}$, B Carapeto ${ }^{1}$, G Rosa $^{1}$, M Cardoso ${ }^{1}$, B Rodrigues ${ }^{1}$, C Matias ${ }^{1}$, C Monteiro ${ }^{1}$, MN Silva ${ }^{1}$, E V Carraça ${ }^{1}$, I Santos ${ }^{1}$, M Pinheiro ${ }^{1}$, H Macias ${ }^{1}$, D Müller ${ }^{1}$, M Costa ${ }^{1}$, J Joaquim ${ }^{1}$, T Gomes ${ }^{1}$, R Francisco ${ }^{1}$, L B Sardinha ${ }^{1}$, P Martins ${ }^{1}$, CS Minderico ${ }^{1}$, and AM Silva

${ }^{1}$ Exercise and Health Laboratory, CIPER, Faculdade de Motricidade Humana da Universidade de Lisboa, Estrada da Costa, 1499-002, Cruz-Quebrada, Portugal

${ }^{2}$ Faculdade de Ciências da Nutrição e Alimentação da Universidade do Porto, Rua Dr. Roberto Frias, 4200-465, Porto, Portugal

Indirect calorimetry (IC) is the gold standard to evaluate resting metabolic rate (RMR) but it requires expensive equipment and consumables and skilled technicians. Alternatively, predictive equations were developed to estimate RMR. In this study we aimed to determine the validity of predictive equations for RMR estimations using IC as the reference method in a sample of former elite athletes. The RMR of fifty-six former athletes (79\% males; age: $42 \pm 9$ y; weight: $95.8 \pm 14.8 \mathrm{~kg}$ ) was measured by IC and predicted using seven equations: Harris-Benedict, Cunningham, Schofield, FAO/WHO/UNU, Owen, Mifflin-St. Jeor, and Katch-McArdle. Dual-energy X-ray densitometry was used to assess fat-free mass. The estimations obtained by the predictive equations overestimated the value measured by the IC, ranging between $70-300 \mathrm{kcal} /$ day $(4-15 \%)$, with percentage values of valid estimations below 59\%. The linear regression between the reference and each of the predictive models did not differ from the identity line with estimated values explaining about $50 \%$ of the variability of the measured values. The agreement between methods was weak for all the equations as observed by the wide limits of agreement. The Harris-Benedict equation was the only model that did not present a significant trend between the differences and the mean of the methods in RMR assessment. Therefore, the overestimation of RMR and the large individual errors observed by the predicted models precludes its use in former elite athletes. New alternative models are required for an accurate estimation of energy requirements at rest for retired elite athletes, specifically if an adequate nutritional intake for weight management purposes is required. 
Effects of Duration of New Zealand Blackcurrant Extract Intake on Whole-Body Fat Oxidation During Moderate Intensity Exercise in Males

\author{
MA Șahin ${ }^{1,2}$, P Bilgiç $^{2}, \mathrm{~S}$ Montanari ${ }^{1}$, and MET Willems ${ }^{1}$ \\ ${ }^{1}$ Institute of Sport, University of Chichester, Chichester, UK \\ ${ }^{2}$ Department of Nutrition and Dietetics, Hacettepe University, Ankara, \\ Turkey
}

New Zealand blackcurrant (NZBC) has a high anthocyanin content. Sevenday intake of NZBC extract enhanced exercise-induced whole-body fat oxidation. We examined the effects of longer intake of NZBC extract (i.e. 14 days) on metabolic and physiological responses during moderate intensity exercise. Sixteen physically active healthy men (mean \pm SD age: $24 \pm 6$ y, body mass: $78 \pm 16 \mathrm{~kg}$, height: $178 \pm 6 \mathrm{~cm}$, BMI: $24.7 \pm 4.2 \mathrm{~kg} \cdot \mathrm{m}^{-2}$, IPAQ score: $4385 \pm 1635 \mathrm{MET} \cdot \mathrm{week}^{-1}$ ) volunteered. In the first visit, resting metabolic equivalent (1-MET) was measured using Douglas bags (1-MET: $\left.3.95 \pm 0.64 \mathrm{ml} \cdot \mathrm{kg}^{-1} \cdot \mathrm{min}^{-1}\right)$. An incremental walking test was performed to determine the relationship between walking speed and MET. A randomised, cross-over experimental design was used. For intake conditions, participants consumed 2 capsules of NZBC extract $(600 \mathrm{mg}$ and containing $210 \mathrm{mg}$ of anthocyanins, CurraNZ ${ }^{\mathrm{TM}}$ Health Currancy Ltd., Surrey, UK) with breakfast with a 14-day washout. On the morning of testing, the final 2 capsules were ingested 2 -hr before the 30-min brisk walk at $4(\mathrm{n}=3)$ or $5(\mathrm{n}=13)$ METs (walking speed: $\left.5.7 \pm 0.7 \mathrm{~km} \cdot \mathrm{h}^{-1}\right)$. Responses were measured at 7-10, 17-20 and 27-30 min during the walk and averaged and analysed with one-way repeated measures ANOVA and post-hoc student t-tests. Duration of intake had no effect on heart rate (baseline: 102 \pm 17 , 7-day: $102 \pm 17$, 14-day: $101 \pm 18$ beats $\cdot \min ^{-1}$ ), minute ventilation (STPD) (baseline 30.3 \pm 5.8 , 7-day: $30.8 \pm 6.3$, 14-day: $30.8 \pm 6.6 \mathrm{~L} \cdot \mathrm{min}^{-1}$ ), oxygen uptake (STPD) (baseline: $1.50 \pm 0.30,7$-day: $1.52 \pm 0.32,14$-day: $1.51 \pm 0.33 \mathrm{~L} \cdot \mathrm{min}^{-1}$ ) and RPE (baseline: $11 \pm 2,7$-day: $11 \pm 2,14$-day: $10 \pm 2$ ). Only 14-day intake resulted in lower RER (baseline: $0.85 \pm 0.05$, 7-day: $0.84 \pm 0.05,14$-day: $0.84 \pm 0.04, \mathrm{P}=0.02$ ) and lower carbohydrate oxidation (baseline: $0.95 \pm 0.40,7$-day: $0.91 \pm 0.40,14$-day: $0.86 \pm 0.33 \mathrm{~g} \cdot \mathrm{min}^{-1}$, $\mathrm{P}=0.03$ ). Fat oxidation was enhanced with 7-day and 14-day intake by $10 \%$ and $16 \%$, respectively (baseline: $0.36 \pm 0.12,7$-day: $0.39 \pm 0.13$, 14-day: $\left.0.41 \pm 0.13 \mathrm{~g} \cdot \mathrm{min}^{-1}, \mathrm{P}=0.007\right)$. It is concluded that 14-day intake of New Zealand blackcurrant extract seems to favour fat more as an energy source during brisk walking compared to 7-day intake. Intake duration of anthocyanin-rich blackcurrant extract may need to be considered when regular moderate intensity exercise is undertaken for body weight management.

Acknowledgement: We thank Health Currancy (UK) Ltd and CurraNZ (NZ) Ltd for supplements and Blackcurrant New Zealand Inc. for support for conference attendance.

\section{Self-Reported Periodisation of Nutrition in Elite Female and Male Runners and Race Walkers}

IA Heikura $^{1,2}$, T Stellingwerff ${ }^{3}$, and LM Burke ${ }^{1,2}$

${ }^{1}$ Mary MacKillop Institute for Health Research, Australian Catholic University, Melbourne, Australia

${ }^{2}$ Australian Institute of Sport, Canberra, Australia

${ }^{3}$ Canadian Sport Institute Pacific, Victoria, Canada

Athletes should achieve event-specific physiological requirements through careful periodisation of training underpinned by individualised and targeted nutrition strategies. However, evidence of whether, and/or how, elite endurance athletes periodise nutrition is scarce. Accordingly, elite international female $(n=67)$ and male $(n=37)$ middle/long-distance athletes (IAAF score:1129 \pm 54 ; corresponds to 13:22.49 [M] and 15:17.93 $[\mathrm{F}]$ in the $5000 \mathrm{~m})$ completed an online survey examining self-reported practices of dietary micro, meso and macro periodisation. Data are shown as the percentage of all athletes practicing a given strategy followed by the $\%$ of athletes reporting various practices within this self-reported strategy. Differences according to sex, event (middle-distance [800/1500m] vs track-distance [3000-10000m] vs road-distance [marathon/race walks]), calibre (major championship qualifier vs the rest), and training volume (low/moderate/high) were analyzed using Chi-square or Kruskal-Wallis test and indicated statistically different when $\mathrm{p} \leq 0.05$. Most athletes reported eating more on hard training days (92\%) and focusing on nutrition before $(84 \%$; carbohydrate [63\%] and timing [58\%]) and after $(95 \%$; protein [59\%], timing [55\%], carbohydrate [50\%]) key sessions. Roaddistance were the most (62\% and 57\%), and middle-distance the least (30\% and $30 \%)$ likely to train fasted $(\mathrm{p}=0.037)$ or restrict carbohydrates periodically $(\mathrm{p}=0.050)$, respectively. Carbohydrate intake during training $(58 \%)$ was more common in road-distance $(90 \%$; $\mathrm{p}<0.001)$ than middle/ track-distance (48\% and 37\%). Most athletes (83\%) reported following a specific diet before and during race day, with $\sim 50 \%$ focusing on carbohydrates. Nearly all (97\%) road-distance athletes reported following a duringrace nutrition plan (carbohydrates/fluids:89\%). Based on our analysis: 1) Road-distance athletes periodise carbohydrate while track/middle-distance avoid low carbohydrate availability; 2) Middle-distance runners emphasize physique goals; 3) Athletes choose nutrition strategies primarily to improve performance, followed by reasons related to physique, adaptation and health. Overall, these athletes appear to possess good knowledge of nutrition for supporting performance.

The Effects of Short-Term High Intensity Interval Training on Resting Metabolic Rate, Substrate Oxidation and Body Composition MM Atakan ${ }^{1}$, ȘN Koșar ${ }^{1}$, Y Güzel ${ }^{1}$, S Bulut $^{1}$, HH Turnagöl ${ }^{1}$

${ }^{1}$ Nutrition and Metabolism in Exercise Division, Faculty of Sport Sciences, Hacettepe University, Turkey

Resting metabolic rate (RMR), substrate oxidation $\left(\mathrm{Sub}_{\mathrm{ox}}\right)$ and body composition (BC) are important health indicators. High-intensity interval training (HIIT) improves BC despite low training volume. Further to this, recent findings over HIIT have revealed that HIIT positively affects a number of health outcomes including increases in cardiorespiratory fitness and maximal fat oxidation, upregulation of skeletal muscle proteins and markers of mitochondrial function related to oxidative phosphorylation capacity and improved body composition. However, there are limited studies on the effects of acute and chronic HIIT undertaken daily twice on $\mathrm{RMR}, \mathrm{Sub}_{\mathrm{ox}}$ and BC. Thus, the aim of this study is to compare the effects of 1 and 2 weeks of HIIT with the same exercise volume on RMR, Sub ${ }_{\text {ox }}$ and BC. Thirty physically active males (age:25 $\pm 4 \mathrm{yr}$ ) were assigned to either the double HIIT (HIIT-D, $\mathrm{n}=15$ ) or the single HIIT (HIIT- S, $\mathrm{n}=13$ ) groups. HIIT-D performed two consecutive training sessions on the same day, separated by $3 \mathrm{~h}$. HIIT-S was trained once per day, 3 days/week for $2 \mathrm{wk}$. Both groups completed 6 HIIT sessions. Each training session consisted of $10^{*} 60 \mathrm{~s}$ repeated efforts of cycling at $\mathrm{VO}_{2 \max }$, interspersed by $75 \mathrm{~s}$ of cycling at low intensity $\left(30 \%\right.$ of $\left.\mathrm{VO}_{2 \max }\right)$. Before and after the training, BC and RMR were measured by dual energy X-ray absorptiometry (DXA) and indirect calorimetry (Cosmed, Italy), respectively. $\mathrm{VO}_{2 \max }$ was measured only once at the beginning of the study to determine the load of HIIT intervention. Sub ${ }_{\text {ox }}$ was calculated using Frayn equation (1983). Repeated measure ANOVA was used to analyze the data. RER decreased in both HIIT-D (pre:0.82 \pm 0.04 ; post:0.80 \pm 0.02 ) and HIIT-S group (pre: $0.83 \pm 0.03$; post: $0.80 \pm 0.03 ; \mathrm{p}<0.05$ ). Accordingly, resting fat oxidation increased in both HIIT-D (pre: $0.07 \pm 0.02 \mathrm{~g} / \mathrm{min}$; post: $0.08 \pm 0.01 \mathrm{~g} / \mathrm{min}$ ) 
and HIIT-S (pre: $0.06 \pm 0.02 \mathrm{~g} / \mathrm{min}$; post: $0.08 \pm 0.02 \mathrm{~g} / \mathrm{min}$ ) while CHO oxidation decreased in both groups (HIIT-D $\mathrm{CHO}_{\mathrm{ox}}=$ pre: $0.14 \pm 0.04 \mathrm{~g} / \mathrm{min}$; post: $0.12 \pm 0.03 \mathrm{~g} / \mathrm{min} ; \mathrm{p}<0.041$; HIIT-S $\mathrm{CHO}_{\text {ox }}=$ pre: $0.17 \pm 0.07 \mathrm{~g} / \mathrm{min}$; post: $0.12 \pm 0.04 \mathrm{~g} / \mathrm{min} ; \mathrm{p}<0.05)$. However, the magnitude of the improvements in $\mathrm{Sub}_{\mathrm{ox}}$ and RER were similar. However, no change was observed in BC or RMR in either group. The short-term HIIT programs (HIIT-D \& HIIT-S) have improved resting $\mathrm{Sub}_{\mathrm{ox}}$ similarly without altering RMR and $\mathrm{BC}$.

\section{Exercise-Induced Dehydration Causes Malabsorption of Carbohydrate Rich Pre-Exercise Meal}

Z Huschtscha ${ }^{1}$, I Russo ${ }^{1}$, RMJ Snipe $^{2}$, D Dixon ${ }^{3}$, V Camões-Costa ${ }^{1,4}$, and RJS Costa ${ }^{1}$

${ }^{1}$ Department of Nutrition Dietetics \& Food, Monash University, Australia

${ }^{2}$ Centre for Sport Research, Deakin University, Australia

${ }^{3}$ Department of Sports Science, Writtle University College, UK

${ }^{4}$ Health \& Aging Research Group, Swinburne University of Technology, Swinburne University of Technology, Australia

It is well established that endurance exercise stress compromises gastrointestinal integrity and functional responses, potentially leading to performance debilitating gastrointestinal symptoms (GIS). However, the magnitude to which exercise-induced dehydration impact gastrointestinal integrity and functional markers is less known. The study aimed to determine the impact of exercise-induced dehydration on gastrointestinal integrity and functional responses, and GIS. Using a randomised crossover design, male endurance runners $(\mathrm{n}=11)$ performed $2 \mathrm{~h}$ of running at $70 \% \dot{V} \mathrm{O}_{2 \max }$ in $25^{\circ} \mathrm{C} \mathrm{T}_{\mathrm{amb}}$ and $46 \% \mathrm{RH}$. On one occasion participants were provided with water $(1689 \pm 542 \mathrm{ml} ; 11.0 \pm 3.5 \mathrm{ml} / \mathrm{kg})$ during exercise (euhydration: EUH; $0.6 \pm 0.6 \%$ body mass loss (BML), $\mathrm{P}_{\text {Osmol }}$ $291 \pm 13 \mathrm{mOsmol} / \mathrm{kg}$, and $\left.\Delta \mathrm{P}_{\mathrm{V}}-1.0 \pm 5.0 \%\right)$, and on another occasion participants were water restricted (dehydration: DEH; 3.1 $\pm 0.7 \% \mathrm{BML}$, $\mathrm{P}_{\text {Osmol }} 306 \pm 11 \mathrm{mOsmol} / \mathrm{kg}$, and $\Delta \mathrm{P}_{\mathrm{V}}-5.8 \pm 3.7 \%$ ). After a $24 \mathrm{~h}$ standardised diet, $2 \mathrm{~h}$ before exercise, participants consumed a standardised mixed carbohydrate breakfast $(731 \mathrm{kcal}, 29 \mathrm{~g}$ protein, $15 \mathrm{~g}$ fat, and $120 \mathrm{~g}$ carbohydrates of which starch $50 \mathrm{~g}$, lactose $15 \mathrm{~g}$, fructose $10 \mathrm{~g}$, dextrose $10 \mathrm{~g}$, sucrose $16 \mathrm{~g}$, maltodextrin $10 \mathrm{~g}$, dietary fibre $6 \mathrm{~g}$, and other FODMAPs $3 \mathrm{~g}$ ), with $700 \mathrm{ml}$ of water provisions. Heart rate, RPE, and GIS were recorded every $15 \mathrm{~min}$ during exercise. Blood samples were collected pre- and post-exercise, and during recovery to determine plasma I-FABP and cortisol. Breath samples were collected every $30 \mathrm{~min}$ in recovery for $3 \mathrm{~h}$ to determine $\mathrm{H}_{2}$ (malabsorption marker) responses. $\mathrm{DEH}$ resulted in higher heart rate $(\mathrm{p}=.038 ; 158 \mathrm{vs} 152 \mathrm{bpm}), \mathrm{RPE}(\mathrm{p}=.001$; 15 vs 13$)$, plasma cortisol ( $\mathrm{p}=.098 ;+286 \mathrm{vs}+176 \mathrm{nMol} / \mathrm{L})$ and I-FABP $(p=.047 ;+539 \mathrm{vs}+371 \mathrm{pg} / \mathrm{ml})$, in response to exertional stress, compared with EUH. A greater AUC for breath $\mathrm{H}_{2}(\mathrm{p}=.026)$ was observed on DEH (1188 ppm/3h; peak +12 ppm) vs. EUH (579 ppm/3h; peak $+6 \mathrm{ppm})$. Despite greater GIS incidence on DEH (82\%) compared with EUH (64\%), severity scores failed to reach significance between trials. DEH compromised absorption of a pre-exercise carbohydrate rich meal more than EUH, possibly through greater intestinal injury and sympathetic drive on DEH. These finding may have implications for post-exercise recovery nutrition tolerance and bioavailability.

\section{New Zealand Blackcurrant Extract Enhances Fat Oxidation During Submaximal Exercise in the Heat}

BJ Lee ${ }^{1}$, TR Flood ${ }^{1}$, AM Hiles ${ }^{1}$, LEV Wheeler ${ }^{1}$, R Costello ${ }^{1}$, EF Walker ${ }^{1}, \mathrm{~K}_{\text {Ashdown }}{ }^{1}$, MR Kuennen ${ }^{2}$, and MET Willems ${ }^{1}$

${ }^{1}$ Institute of Sport, University of Chichester, Chichester, UK
${ }^{2}$ Department of Exercise Science, High Point University, High Point, North Carolina, USA

Supplementation for 7 days with anthocyanin rich New Zealand blackcurrant (NZBC) extract has been shown to increase fat oxidation in healthy men and women during submaximal exercise under neutral climatic conditions. We investigated whether alterations in substrate metabolism would be observed while exercising in hot climatic conditions after 7 days intake of NZBC extract. In a randomized double-blind design, 12 men and 6 women (mean \pm SD: Age $27 \pm 6$ years, height $1.76 \pm 10 \mathrm{~m}$, mass $74 \pm 12 \mathrm{~kg}, \mathrm{VO}_{2 \max } 53.4 \pm 7.0 \mathrm{~mL} \mathrm{~kg}^{-1} \cdot \mathrm{min}^{-1}$ ) completed 4 experimental visits after an overnight fast. Following a preliminary assessment and familiarization session, participants ingested $2 \times 300 \mathrm{mg} \mathrm{day}^{-1}$ capsules of CurraNZ $^{\mathrm{TM}}$ (each containing $105 \mathrm{mg}$ anthocyanin) or a visually matched placebo ( 2 x $300 \mathrm{mg}$ microcrystalline cellulose M102) for 7 days (washout 14 days). On day 7, participants performed 60 minutes of running at $65 \%$ $\mathrm{VO}_{2 \max }$ in hot ambient conditions $\left(34^{\circ} \mathrm{C}\right.$ and $40 \%$ relative humidity). Women completed the experimental trials in the mid-luteal phase of the menstrual cycle, verified by cycle length tracking, ovulation prediction testing, and post-hoc progesterone concentrations. Substrate oxidation was determined via indirect calorimetry. Data are presented as mean and 95\% confidence intervals. NZBC extract had no effect on heart rate (PL: 173 (157 to 190$)$ ) NZBC: 174 (156 to 192) beats $\mathrm{min}^{-1}$ ) and oxygen uptake (PL: 2.74 (1.73 to 3.76 ); NZBC: 2.78 (1.78 to 3.79$) \mathrm{L} \cdot \mathrm{min}^{-1}$. NZBC extract increased fat oxidation by $30 \%$ compared to placebo (PL: 0.54 (0.18 to 0.90); NZBC: 0.69 (0.31 to 1.07$\left.) \mathrm{g} \cdot \mathrm{min}^{-1}, \mathrm{p}<0.01, d=0.58\right)$. Carbohydrate oxidation was decreased by $8 \%$ during the NZBC trial (PL: 2.19 (1.06 to 3.31 ), NZBC: 1.92 (0.88 to 2.96$\left.) \mathrm{g} \cdot \mathrm{min}^{-1}, \mathrm{p}<0.05, \mathrm{~d}=0.46\right)$. These results are reflected in substrate utilization data, with carbohydrate oxidation decreased by $47 \mathrm{kcal}(95 \% \mathrm{CI}: 27-69 \mathrm{kcal}, \mathrm{p}<0.05, \mathrm{~d}=0.31)$, and fat oxidation increased by $63 \mathrm{kcal}(95 \% \mathrm{CI}: 36-90 \mathrm{kcal}, \mathrm{p}<0.01, \mathrm{~d}=0.50)$. With NZBC, RER was 0.02 units lower (PL: 0.89 (0.82 to 0.97); NZBC: 0.85 ( 0.77 to 0.98$), \mathrm{p}<0.05, \mathrm{~d}=0.06$. It is concluded that seven days intake of $600 \mathrm{mg}$ New Zealand blackcurrant extract increased fat oxidation during submaximal treadmill running in hot conditions.

Acknowledgement: We thank Health Currancy (UK) Ltd and CurraNZ (NZ) Ltd for supplements and Blackcurrant New Zealand Inc. for support for conference attendance.

\section{Effects of 'Night Time Recharge' Sleep Supplement on Sleep in Active Young Adults}

T Simper ${ }^{1}$, and M Gilmartin ${ }^{1}$

${ }^{1}$ Food and Nutrition Group, Sheffield Hallam University, Sheffield South Yorkshire, UK

Concentrated Cherry juice and products containing cherry have been purported to improve sleep efficiency. In this investigation we sought to verify the effects of a product containing cherry active on various sleep parameters (Time in bed, total sleep, sleep efficiency, sleep latency) in young active adults. 20 people ( 9 female; median 21 y, IQR 20-21) took part in a double blind randomised control trial with crossover. GENEActiv accelerometers were used to assess physical activity levels. 7-day diet diaries were recorded to assess key dietary factors likely to affect sleep. Urinary levels of 6-sulfatoxymelatonin (6-SMT) were assessed via Enzyme Linked Immunosorbent Assay (ELISA). Participants had similar (not statistically different) levels of physical activity and dietary intake for both the supplement and placebo arms of the trial. The median average 6-SMT for placebo was $4 \mathrm{ng} / \mathrm{ml}$ (IQR: 2-4 ng/ml) and $29 \mathrm{ng} / \mathrm{ml}$ (IQR: $21-30 \mathrm{ng} / \mathrm{ml}$ ) for the supplement $(P<0.001)$. No significant difference occurred in relation to our main outcome measure (sleep efficiency) despite the clear 
difference in the presence of the key metabolite, melatonin (6-SMT). Furthermore, participants found the taste of the supplement disagreeable and also felt that they fell asleep more easily with the supplement than the placebo. There was a slight difference in tryptophan intake during the supplement arm and this was statistically significantly greater than the placebo (although the expected effect of this would be improved sleep outcomes which did not occur). Further investigations into the possible effects of cherry derivatives on sleep latency should be carried out with participants who have problems specifically with sleep onset. This study is limited by a small number of participants and may have benefited from corroborating methods of measuring sleep quality, for example a validated sleep questionnaire. This is the first, to our knowledge, study carried out on Night Time Recharge.

\section{Are Fruit Juices Healthier Than Sweetened Beverages? New Perspectives}

A Pépin ${ }^{1}, \mathrm{KL}$ Stanhope ${ }^{2}$, and P Imbeault ${ }^{1}$

${ }^{1}$ Behavioural and Metabolic Research Unit, University of Ottawa, Canada

${ }^{2}$ Department of Molecular Biosciences, University of California (Davis), USA

The consumption of sweetened beverages is associated with increased calorie intake, long-term weight gain and increased prevalence of risk factors for metabolic diseases such as an abnormal elevation of blood lipids like circulating cholesterol and triglycerides. The evidence strongly suggests that free sugars, especially fructose, comprised in beverages sweetened with High-Fructose Corn Syrup is responsible for altering blood lipids. $100 \%$ natural fruit juices have a fructose composition very similar to sweetened beverages. Thus, could fruit juice consumption lead to the same adverse effects on health as sweetened beverages? We searched in PubMed using the terms "fruit juice", "sweetened beverages", "fructose" and "High-Fructose Corn Syrup". The complete reference lists of all retrieved papers were further searched. Included articles were in English, with no limit with regards to the year of publication and reported lipid outcomes. A total of 6 meta-analyses and systematic reviews, 24 reviews, 9 epidemiological or population studies, 3 animal or in vitro studies, 38 trials and 16 other documents (public guidelines, government statistics, etc.) were identified and included in our review. Nearly all of the retrieved studies investigated a dose of free sugars from fruit juices well below the average consumption estimates, very few directly compared the effects of fruit juices to sweetened beverages and none controlled for diet variations. Despite the similarity of fruit juices to sweetened beverages in terms of fructose content, it remains unclear whether they lead to the same metabolic consequences. Important discrepancies in the current literature makes it impossible to provide evidence-based public recommendations as to whether the consumption of fruit juices alters the blood lipid profile. More RCTs comparing the metabolic effects of fruit juice and sweetened beverage consumption under dietary conditions that will eliminate confounding by weight gain and by diet differences are needed in order to shape accurate public health guidelines on the variety and quantity of free sugars in our diet that would help to prevent the development of metabolic diseases.

\section{Revisiting the Exclusion of the Initial Five Min of Indirect Calorimetry Measurement of RMR}

\footnotetext{
J Heydenreich $^{1,2}$, B Kayser ${ }^{2}$, Y Schutz ${ }^{3}$, and K Melzer ${ }^{1}$

${ }^{1}$ Swiss Federal Institute of Sport, Magglingen, Switzerland

${ }^{2}$ Institute of Sport Sciences, University of Lausanne, Lausanne, Switzerland
}

${ }^{3}$ Department of Physiology, University of Fribourg, Fribourg, Switzerland

Most guidelines for the measurement of resting metabolic rate (RMR) with indirect calorimetry recommend at least 20 min supine rest prior to measurement but also to discard the initial five minutes of measurement. Purpose of this study was to measure RMR in men and women endurance trained athletes $(\mathrm{ATH})$ and healthy controls $(\mathrm{CON})$ and to compare two RMR data analysis methods, with and without deletion of the initial five min. RMR was measured for 30 consecutive min after an overnight fast using indirect calorimetry in 58 women $(29 \mathrm{ATH}, 29 \mathrm{CON} ; 28.2 \pm 5.1 \mathrm{y}$, $\left.21.2 \pm 1.6 \mathrm{~kg} / \mathrm{m}^{2}\right)$ and 56 men $(31 \mathrm{ATH}, 25 \mathrm{CON} ; 26.6 \pm 4.9 \mathrm{y}, 22.3 \pm$ $2.0 \mathrm{~kg} / \mathrm{m}^{2}$ ). Subjects rested for $30 \mathrm{~min}$ before the measurement started. For calculating RMR, the five-min-interval with the lowest mean Coefficient of Variation (CV) for $\mathrm{VO}_{2}$ and $\mathrm{VCO}_{2}$, a CV of $<10 \%$ for $\mathrm{VO}_{2}$ and $\mathrm{VCO}_{2}$, and a Respiratory Quotient $\geq 0.70$ and $\leq 1.00$ was searched. According to the recommendations we discarded the initial five min and searched for the interval in the remaining $25 \mathrm{~min}$ (RMRbest25). In addition, the interval was also searched in the complete observation time including the initial five min (RMRbest30). $T$-tests for independent samples were used for RMR sex comparisons. For comparison of RMRbest 25 and RMRbest30 $t$ tests for dependent samples were used $(\alpha=.05)$. RMRbest 25 was significantly higher in men compared to women $(1847 \pm 194 \mathrm{vs.} 1482 \pm 151 \mathrm{kcal} /$ d; $p<.0001$ ). In $44.8 \%$ (ATH: $n=12, \mathrm{CON}: n=14$ ) of women and $21.4 \%$ (ATH: $n=3$, CON: $n=9$ ) of men the five-min interval with the lowest mean CV started within the initial five min of RMR measurement. RMRbest30 was significantly lower than RMRbest 25 in women $(1417 \pm 112$ vs. $1441 \pm 194 \mathrm{kcal} / \mathrm{d} ; p<.05)$, but not in men $(1852 \pm 157$ vs. $1841 \pm 148 \mathrm{kcal} / \mathrm{d} ; p=.62)$. Based on the $\mathrm{CV}$ criteria there were no reasons to fully discard the initial five min. The lower RMR when taking the initial five min into account can be an artifact or represent a best estimate. We recommend not to discard systematically the initial five min period of indirect calorimetry, when subjects are properly rested before RMR measurement. Researchers should carefully screen the complete period of measurement (visual inspection) for each subject and objectively decide, based on the data profile, which five min interval should be used for subsequent analysis as well as report their methods of data analysis in detail.

\section{Comparison of the Effects of Tart Cherry Juice and Pomegranate Juice on Exercise-Induced Muscle Damage and Recovery}

A Lynn ${ }^{1}$, KL. Lamb ${ }^{1}$, E Johnson ${ }^{2}$, and M Ranchordas ${ }^{3}$

${ }^{1}$ Food and Nutrition Group, Sheffield Hallam University, Sheffield, UK

${ }^{2}$ Department of Oncology, Human Nutrition, University of Sheffield, Sheffield, UK

${ }^{3}$ Academy of Sport and Physical Activity, Sheffield Hallam University, Sheffield, UK

Drinks rich in polyphenols have been shown to enhance recovery after exercise. Studies investigating the independent effects of tart cherry juice or pomegranate juice have demonstrated reductions in exercise-induced muscle soreness and enhanced recovery. However, no study has directly compared cherry juice with pomegranate juice, therefore, the purpose of this study was to compare the effects of both juices on recovery from exercise-induced muscle damage. A total of 27 non-resistance trained men (age 28 y (SD 9.5); BMI $25.5 \mathrm{~kg} / \mathrm{m}^{2}$ (SD 4.0)) were recruited to a randomised, placebo-controlled, 3-arm, parallel design study. Participants consumed two servings of pomegranate juice $(250 \mathrm{~mL}$ per serving), tart cherry $(30 \mathrm{~mL}$ of concentrate diluted to $250 \mathrm{~mL}$ ) or a fruit flavoured placebo drink $(250 \mathrm{~mL})$ for $9 \mathrm{~d}$; all drinks were matched for energy through the addition of maltodextrin. On day 5, participants completed 5 sets of 
10 single arm maximal eccentric contractions of the elbow flexors. Maximal isometric voluntary contractions (MIVC) were measured preexercise, immediately after exercise and 24 h, 48 h, $72 \mathrm{~h}$ and $96 \mathrm{~h}$ later. Whole blood creatine kinase (CK), pain pressure threshold (PPT), range of motion (ROM) and delayed onset of muscle soreness (DOMS) were also measured at all time points. All participants consumed a standardised meal immediately after the exercise challenge and were asked to avoid antioxidant supplements and polyphenol rich foods for the duration of the study. The data were analysed using mixed model ANOVA. The exercise protocol invoked significant decreases in MIVC $(\mathrm{P}<0.001$; max decrease of $26.5 \%, 24 \mathrm{~h}$ post-exercise), PPT ( $\mathrm{P}=0.001$; max decrease of $20.7 \%$, $48 \mathrm{~h}$ post-exercise $)$ and $\mathrm{ROM}(\mathrm{P}=0.001$; $\max$ decrease of $5.6 \%, 72 \mathrm{~h}$ postexercise) and significant increases in $\mathrm{CK}(\mathrm{P}=0.04$; max increase $967 \%$, $96 \mathrm{~h}$ post-exercise) and DOMS ( $\mathrm{P}<0.001$; max increase $34.8 \%, 48 \mathrm{~h}$ postexercise) indicating that substantial muscle damage occurred. However, the responses were similar in each group for all outcome measures and there were no statistically significant differences between groups (main effect of group, $\mathrm{P}>0.05$ or group $\mathrm{x}$ time interaction $\mathrm{P}>0.05)$. These data suggest that in non-resistance trained men neither pomegranate juice nor tart cherry juice enhance recovery from high force eccentric exercise of the elbow flexors.

\section{A Pilot Study Using Continuous Blood Glucose Monitoring During Mountain Ultra-Marathon}

E Mori $^{1}$, and K Ishihara ${ }^{1}$

${ }^{1}$ Dept of Food Science and Human Nutrition, Ryukoku University, Japan

The present study was to measure the variability of blood glucose during over $100 \mathrm{~km}$ mountain marathon using continuous glucose monitoring system. The relationships among blood glucose, elevation gain, and subjective feelings of fatigue were investigated in 2 elite ultramarathon runners. One runner (runner A) participated in the race of $104 \mathrm{~km}$ and $5,239 \mathrm{~m}$ elevation gain and finished in $16.3 \mathrm{~h}$ and the other (runner B) participated in the race of $160.3 \mathrm{~km}$ and $8,381 \mathrm{~m}$ elevation gain and finished in $29.4 \mathrm{~h}$. The average blood glucose was $150 \pm 30$ and $142 \pm 19$ in runner A and $\mathrm{B}$, respectively. There observed no relationships between elevation gain and blood glucose change in both runners. Post-race survey exhibited significant decreases of blood glucose in the sections where subjective feelings of fatigue were recorded in both runners, compared to the rest of the sections $(-15.9 \pm 11$ and $-10.8 \pm 6.4$ in runner $\mathrm{A}$ and $\mathrm{B}$, respectively). M-value, an index of glucose variability, was less than 5 for the first $9.5 \mathrm{~h}$, which indicated good glycemic control $(\mathrm{M}$-value $<5.0)$ in both runners. On the other hand, rapid rise and falls, outliners of M-value were frequently observed in the last quarter of the race in both runners. These findings suggest that modest glycemic control was difficult during the last $25 \%$ of the race time though these 2 elite runners regularly ingested supplementary food and drink at a constant pace.

\section{Pre-Season Dietary Habits of Indian Competitive Age Group Swimmers}

M Khopkar ${ }^{1}$

${ }^{1}$ Department of Foods, Nutrition \& Dietetics, Nirmala Niketan, College of Home Science, Mumbai, Maharashtra, India

It is important for swimmers in the pre-competition season to consume high quality and adequate quantity of energy and macronutrients for maximum training adaptations. The aim of this study was to determine the pre-season dietary habits of state, national and international swimmers aged 13-17y from different parts of India. Twenty-seven competitive swimmers were selected via purposive sampling. Anthropometric, body composition and training details were recorded. Dietary intake was assessed using a 3day diet recall to determine the quality of pre-duringpost swim meals, supplement intake and quantity of energy and macronutrients consumed. These were further compared with total energy expenditure; calculated through predictive equations and standard literature recommendations. It was found that $70 \%$ swimmers adhered to the $1-2 \mathrm{~h}$ pre-training meal guidelines, however none of the swimmers followed the carbohydrate plus protein rich meal immediately post training sessions. Nearly all (89\%) swimmers consumed at least one supplement with majority $(74.1 \%)$ consuming whey protein. The daily energy intake was found to be lower than the total energy expenditure in $74 \%$ swimmers. Also, both male [t $(15)=-4.703, \mathrm{p}=0.000]$ and female $[\mathrm{t}(10)=-3.438$, $\mathrm{p}=0.006]$ swimmers consumed lower than recommended daily energy, which also reflected in all swimmers complaining of either one of the following; fatigue/poor recovery/lack of strength \& power. In males, daily protein intake $[\mathrm{t}(15)=2.96, \mathrm{p}=0.010)$ and in both males $[\mathrm{t}(15)=3.94$, $\mathrm{p}=0.001]$ and females $[\mathrm{t}(10)=3.22, \mathrm{p}=0.009]$ daily carbohydrate intake was found to be higher than recommended. The findings of the study suggest that the dietary intakes of competitive age group swimmers do not fully meet the recommendations. Dietary intakes should therefore be optimized and customized for maximum adaptations to pre-season training loads.

\section{Day 2-Posters}

\section{New Zealand Blackcurrant Extract Attenuates Enterocyte Damage Following Exertional Heat Stress}

BJ Lee ${ }^{1}$, TR Flood ${ }^{1}$, AM Hiles ${ }^{1}$, LEV Wheeler $^{1}$, R Costello ${ }^{1}$, EF Walker $^{1}$, K Ashdown ${ }^{1}$, MET Willems ${ }^{1}$, and MR Kuennen ${ }^{2}$

${ }^{1}$ Institute of Sport, University of Chichester, Chichester, UK

${ }^{2}$ Department of Exercise Science, High Point University, High Point, North Carolina, USA

Exertional-heat stress has the potential to disturb intestinal integrity and disrupt intestinal epithelial cell tight junction proteins resulting in enterocyte damage and increased permeability to luminal endotoxins, increasing the risk of heat stroke. Over the past decade at least nine different dietary supplements have been tested for potential improvements in gastrointestinal barrier function during exercise-heat stress, with polyphenolic compounds showing the most promise in preserving intestinal integrity. This study investigated the effects of 7 days of $600 \mathrm{mg}$ New Zealand Blackcurrant intake on intestinal fatty acid binding protein (I-FABP) responses following an acute period of exertional heat stress. In a randomized double blind, cross-over design, 12 un-acclimatized men (Age: 286 years, stature: $1.81 \pm 0.07 \mathrm{~m}$, mass: $80.5 \pm 9.8 \mathrm{~kg}$, body surface area: $2.01 \pm 0.15 \mathrm{~m}^{2}$, sum of 4 skinfold measurements: $32.2 \pm 10.4 \mathrm{~mm}$, maximal aerobic capacity $55.6 \pm 6.0 \mathrm{~mL} \mathrm{~kg}^{-1} \mathrm{~min}^{-1}$ ) completed 4 experimental visits after an overnight fast. Participants ingested 2 × $300 \mathrm{mg}^{- \text {day }^{-1}}$ capsules of CurraNZ ${ }^{\mathrm{TM}}$ (each containing $105 \mathrm{mg}$ anthocyanin) or a visually matched placebo ( 2 x $300 \mathrm{mg}$ microcrystalline cellulose M102) for 7 days (washout 14 days). On day 7, participants performed 60 minutes of running at $65 \% \mathrm{VO}_{2 \max }$ in hot ambient conditions $\left(34^{\circ} \mathrm{C}\right.$ and $40 \%$ relative humidity). Intestinal fatty acid binding protein was measured in citrate plasma at rest, after exercise, and 60 and 240 minutes after exercise as a marker of enterocyte damage. Post exercise I-FABP measurements were lower in the NZBC trial at 20 minutes [by $146 \mathrm{pg}^{-1} \mathrm{~mL}^{-1}(95 \% \mathrm{CI}: 60-231$ $\left.\mathrm{pg} \cdot \mathrm{mL}^{-1}\right) ; \mathrm{t}(11)=3.8, \mathrm{p}=0.003, \mathrm{~d}=0.83$ ], 60 minutes [by $158 \mathrm{pg}^{-\mathrm{mL}^{-1}}$ $\left.\left(95 \% \mathrm{CI}: 95-221 \mathrm{pg} \cdot \mathrm{mL}^{-1}\right) ; \mathrm{t}(11)=5.5, \mathrm{p}=0.002, \mathrm{~d}=1.37\right]$ and 4 hours [by $117 \mathrm{pg} \mathrm{mL}^{-1}\left(95 \% \mathrm{CI}: 50\right.$ to $\left.185 \mathrm{pg}^{-1}\right), \mathrm{t}(11)=3.8, \mathrm{p}=0.003$, $\mathrm{d}=1.65$ ] post exercise compared with equivalent time points after the 
placebo trial. The effect sizes and p-values equate to a false positive risk of $<4 \%$ for all post exercise I-FABP measurements, indicating robust observations with a low false positive risk. It is concluded that 7 days of intake of $600 \mathrm{mg}$ New Zealand blackcurrant extract attenuates enterocyte damage following exertional heat stress. Our findings have implications for athletes training and competing in hot environments.

\section{Hydrogel Carbohydrate Electrolyte Beverage Does Not Improve Blood Glucose Availability, Carbohydrate Malabsorption, Gastrointestinal Symptoms or Carbohydrate Oxidation During Endurance Running, or Time to Exhaustion Performance Test, Compared to a Standard Concentration and Nutrient-Matched Beverage}

AJ McCubbin ${ }^{1}$, A Zhu ${ }^{1}$, S Gaskell, and RJS Costa ${ }^{1}$

${ }^{1}$ Dept of Nutrition, Dietetics \& Food, Monash University, Australia

Ingesting concentrated carbohydrate $(\mathrm{CHO})$ solutions during endurance running can contribute to gastrointestinal symptoms (GIS), due to reduced gastric emptying and/or bacterial fermentation of malabsorbed CHO. A CHO-electrolyte beverage that produces a $\mathrm{pH}$-sensitive hydrogel (HYD) claims to reduce GIS by increasing gastric emptying and intestinal absorption. The aim of this study was to determine if HYD improves blood glucose (BG) availability, CHO malabsorption, GIS, total $\mathrm{CHO}$ oxidation (CHOOx), and time to exhaustion (TTE) following endurance running, compared to a nutrient-matched placebo (PLA). Eight trained male runners (mean $\pm \mathrm{SD}$ : age $35 \pm 5 \mathrm{y}$, weight $75 \pm 4 \mathrm{~kg}, \dot{V} \mathrm{O}_{2 \max } 59 \pm$ $8 \mathrm{~mL} / \mathrm{kg} / \mathrm{min}$ ) completed a $3 \mathrm{~h}$ steady state run at $60 \% \dot{V} \mathrm{O}_{2 \max }$, consuming HYD or PLA ( $90 \mathrm{~g} / \mathrm{h} \mathrm{CHO}, 16 \% \mathrm{w} / \mathrm{v}$ solution) in a double-blind randomised crossover design. They then completed a TTE test of 3 min increments at increasing speed. There was no effect of trial on pre-exercise body mass or total body water, or body mass loss during running. There was an effect of time ( $\mathrm{p}=0.025)$, but not trial on BG concentration measured every 30 min during running. $\mathrm{BG}$ area under the curve was not different between trials (HYD $1091 \pm 97 \mathrm{mmol} / \mathrm{L} / 3 \mathrm{~h}$, PLA $1076 \pm 62 \mathrm{mmol} / \mathrm{L} / 3 \mathrm{~h} ; \mathrm{p}=0.486$ ). CHO malabsorption assessed by breath hydrogen $\left(\mathrm{H}_{2}\right)$ was greater for HYD at $45 \min ($ HYD $8 \pm 11 \mathrm{ppm}$, PLA $4 \pm 5 \mathrm{ppm} ; \mathrm{p}=0.043$ ) and $60 \mathrm{~min}$ (HYD $9 \pm 9$ ppm, PLA $5 \pm 7$ ppm; p=0.046) post-exercise. Only two participants during HYD and one during PLA exceeded the clinically significant $\mathrm{H}_{2}$ threshold of 10 ppm. GIS incidence was $100 \%$ in both trials, but only one participant experienced severe GIS during HYD and two during PLA. Total GIS (summative score: HYD $26 \pm 33$, PLA $29 \pm 31$; $\mathrm{p}=0.397$ ) and gut discomfort (HYD 13 \pm 14 , PLA $15 \pm 13 ; \mathrm{p}=0.462$ ) were not different between trials. CHOOx after $3 \mathrm{~h}$ was not different between trials at $73 \% \dot{V} \mathrm{O}_{2 \max }(\mathrm{HYD} 2.4 \pm 0.5 \mathrm{~g} / \mathrm{min}$, PLA $2.3 \pm 0.9 \mathrm{~g} / \mathrm{min}$; $\mathrm{p}=0.575), 81 \% \dot{V} \mathrm{O}_{2 \max }(\mathrm{HYD} 3.9 \pm 0.9 \mathrm{~g} / \mathrm{min}$, PLA $3.4 \pm 0.6 \mathrm{~g} / \mathrm{min} ; \mathrm{p}=$ 0.091 ) or $91 \% \dot{V} \mathrm{O}_{2 \max }$ (HYD $5.4 \pm 1.6 \mathrm{~g} / \mathrm{min}$, PLA $5.0 \pm 0.8 \mathrm{~g} / \mathrm{min} ; \mathrm{p}=$ 0.500). TTE was not different between trials (HYD 12.0 $\pm 3.2 \mathrm{~min}$, PLA $12.7 \pm 3.3 \mathrm{~min} ; \mathrm{p}=0.066)$. We conclude that consumption of HYD $(90 \mathrm{~g} / \mathrm{h}$ $\mathrm{CHO}, 16 \% w / v)$ does not influence GIS, BG availability, or CHO malabsorption during endurance running, and does not improve $\mathrm{CHOOx}$ and TTE during incremental intensity running until exhaustion.

Intra-individual Responses to New Zealand Blackcurrant Extract During High-Intensity, Intermittent Running: A Repeat Response Study

IC Perkins ${ }^{1}$, SD Blacker ${ }^{1}$, and MET Willems ${ }^{1}$

${ }^{1}$ Institute of Sport, University of Chichester, UK

Intake of New Zealand blackcurrant (NZBC) extract for 7 days improved high-intensity intermittent running (HIIR) performance. This study examined the repeat response of NZBC extract on HIIR. Sixteen active males (age: $23 \pm 3$ y, height: $179 \pm 5 \mathrm{~cm}$, mass: $79 \pm 11 \mathrm{~kg}, \dot{V} \mathrm{O}_{2 \max }: 55.3$ $\pm 5 \mathrm{~mL} \cdot \mathrm{kg}^{-1} \cdot \mathrm{min}^{-1}$, velocity at $\dot{V} \mathrm{O}_{2 \text { max }}: 17.2 \pm 0.8 \mathrm{~km} \cdot \mathrm{h}^{-1}$, mean $\pm \mathrm{SD}$ ) participated. In visit 1 , a ramp protocol was completed to establish $\dot{V} \mathrm{O}_{2 \max }$ and velocity at $\dot{V} \mathrm{O}_{2 \max }$, to individualise exercise intensity for the HIIR test. In visits 2-5, familiarised subjects completed the HIIR test with each stage consisting of six $19 \mathrm{~s}$ high-intensity running bouts, interspersed by $15 \mathrm{~s}$ of low-intensity running, followed by 1 minute of rest. This was repeated at increasing speeds until exhaustion. Prior to visits 2-5, subjects consumed two times capsulated NZBC extract $\left(600 \mathrm{mg} \cdot \mathrm{day}^{-1} \mathrm{CurraNZ}^{\mathrm{TM}}\right.$; containing $210 \mathrm{mg}$ anthocyanin) or two times placebo (P) for 7 days (double blind, randomised, cross-over design, wash-out $\geq 14$ days). Performance was analysed using one-way ANOVA with post-hoc one-tailed t-tests. Blood lactate, heart rate, oxygen uptake and RPE for stages 1-3 were analysed using two-way ANOVAs. Significance was accepted at $\mathrm{p}<0.05$. NZBC increased total running distance by 5.6-7.9\% (NZBC1: $4991 \pm 952 \mathrm{~m}$, NZBC2: $4936 \pm 688 \mathrm{~m}, \mathrm{P} 1:$ 4597 $\pm 806 \mathrm{~m}, \mathrm{P} 2: 4674 \pm 973 \mathrm{~m})$, with NZBC conditions higher than placebo [NZBC1 vs P1 $(\mathrm{p}<0.01)$ and $\mathrm{P} 2, \mathrm{p}=0.01)$, NZBC2 vs PL1 $(\mathrm{p}<0.01)$ and P2 $(\mathrm{p}<0.05)]$. Distance covered during high-intensity running increased by $6.8-10 \%$ with NZBC (NZBC1: 2651 \pm 617 m, NZBC2: $2610 \pm 451 \mathrm{~m}, \mathrm{P} 1: 2386 \pm 510 \mathrm{~m}, \mathrm{P} 2: 2444 \pm 608 \mathrm{~m})$ with NZBC conditions higher than placebo $[\mathrm{NZBC} 1$ vs $\mathrm{P} 1(\mathrm{p}<0.01)$ and $\mathrm{P} 2$ $(\mathrm{p}<0.01)$, NZBC2 vs PL1 $(\mathrm{p}<0.01)$ and P2 $(\mathrm{p}<0.05)]$. Nine participants improved total distance by $\geq 7.9 \%$ in at least one NZBC trial. Six participants $(\sim 38 \%)$ improved total distance by $\geq 8.3 \%$ (mean \pm SD: 17.5 $\pm 7.0 \%$, range: $8.3-37.8 \%$ ) on both NZBC trials. Heart rate, lactate, RPE and oxygen uptake were not different between conditions for stages 1-3. It is concluded that NZBC extract may consistently enhance high-intensity intermittent running performance, but some intra-individual differences are present. These findings may have implications for nutritional interventions in team sports characterised by high-intensity intermittent exercise.

Acknowledgement: We thank Health Currancy (UK) Ltd and CurraNZ (NZ) Ltd for supplements and Blackcurrant New Zealand Inc. for support for conference attendance.

\section{The Effect of an Acute Bout of Rugby Training on Plasma} Concentrations of Intestinal Fatty Acid Binding Protein

S Chantler ${ }^{1}$, B Jones ${ }^{1,4}$, G. Roe ${ }^{1,2}$, P Phibbs ${ }^{1}$, C Owen ${ }^{1}$, A Griffiths ${ }^{1}$, C Ramirez-Lopez ${ }^{1}$, G Davison ${ }^{3}$, and K Deighton ${ }^{1}$

${ }^{1}$ Institute for Sport, Physical Activity \& Leisure, Leeds Beckett University, England

${ }^{2}$ Bath Rugby Club, Bath, UK

${ }^{3}$ School of Sport and Exercise Sciences, University of Kent

${ }^{4}$ Yorkshire Carnegie RUFC, Leeds, UK

Exercise appears to elicit damage to the gut endothelial cell lining, as demonstrated by elevated plasma concentrations of intestinal fatty acid binding protein (iFABP). However, the majority of research to date has focussed on responses to endurance exercise, with little understanding of the potential impact of contact-based sports on gastrointestinal damage. The high intensity nature of rugby, combined with trauma from collisions, suggests that this may cause considerable increases in gastrointestinal damage and permeability. The aim of this study was to investigate the effects of a standardised rugby training session on plasma concentrations of iFABP as a marker of gastrointestinal damage. Nineteen male academy level rugby players $(($ mean \pm SD) age $20 \pm 2$ years, weight; $100.1 \pm$ $13.9 \mathrm{~kg}$ ) completed a standardised rugby training session at the beginning of the pre-season period. Participants arrived after an overnight fast after abstaining from spicy food, alcohol, caffeine and exercise in the previous 
24-hours. All participants were declared fit to play and reported no gastrointestinal illness or disturbances. A venous blood sample and gastrointestinal symptoms were collected immediately before and after the exercise test. The exercise session was based on a four-person rugbybased situational practice, with an emphasis on evasion skills, collision and within-contact exertion (contact-based conditioning). Each group of participants rotated through four different roles; one attacking, one defending, and two as supporting defenders. Each block was approximately 8 minutes in duration and was repeated 3 times for a total of 24 minutes. Plasma concentrations of iFABP increased significantly from $2024 \pm 913$ before the training session to $3624 \pm 1790 \mu \mathrm{g} / \mathrm{ml}$ after the training session ( $\mathrm{p}=$ 0.003 ). However, there were minimal gastrointestinal symptoms reported before or after exercise. In conclusion, a standardised rugby training session as part of pre-season training significantly increased plasma concentrations of iFABP, which is indicative of gastrointestinal damage. Further research is required to establish whether this damage is sufficient to increase gastrointestinal permeability as this may have consequences for bacterial translocation, inflammation and nutrient absorption during recovery.

\section{The Effect of Carbohydrate Intake, Below and Above Current Guidelines Using a Novel Sports Drink, on 3h Endurance Cycling Performance} B Price ${ }^{1}$, A Cheruiyot ${ }^{1}, S$ Sutehall ${ }^{2,3}$, Y Pitsiladis ${ }^{3}$, and S Galloway ${ }^{1}$
${ }^{1}$ Faculty of Health Sciences \& Sport, University of Stirling, Stirling,
UK

${ }^{2}$ Division of Exercise Science and Sports Medicine, University of Cape Town, Cape Town, South Africa

${ }^{3}$ University of Brighton, Welkin House, Eastbourne, UK

In a randomised, repeated measures, single blind, crossover design, 11 active male cyclists, age $37 \pm 11 \mathrm{y}$, height $180 \pm 5 \mathrm{~cm}$, mass $72.4 \pm 3.3 \mathrm{~kg}$, peak power output $384 \pm 36$ Watts, VO2 max 55.6 \pm 6.8 ml.kg-1.min-1, completed a familiarisation and 4 experimental trials consisting of 150 minutes steady state cycling at 95\% lactate threshold (80-90rpm) before performing a work-matched $(\sim 30 \mathrm{~min})$ time trial $(\mathrm{TT})$ task. Participants replicated 48-hour dietary intake and consumed a standardised $1.2 \mathrm{~g}$ carbohydrate $(\mathrm{CHO}) / \mathrm{kg}$ body mass breakfast before each trial. During each trial participants consumed a flavour and sodium matched beverage providing $\mathrm{CHO}$ at either $0 \mathrm{~g} / \mathrm{h}, 45 \mathrm{~g} / \mathrm{h}$ glucose, $90 \mathrm{~g} / \mathrm{h}$ maltodextrin:fructose, or a novel alginate containing sports drink providing $135 \mathrm{~g} / \mathrm{h}$. Glucose, lactate, RPE, heart rate, gastrointestinal (GI) distress and expired gases were recorded at intervals during the steady state exercise. A mean difference in TT times of $90 \mathrm{~s}$ (95\% CI +16 s to $-197 \mathrm{~s}), 153 \mathrm{~s}$ (95\% CI $-37 \mathrm{~s}$ to $-270 \mathrm{~s}$ ) and $150 \mathrm{~s}(95 \% \mathrm{CI}+80$ s to $-381 \mathrm{~s}$ ) was observed between $0 \mathrm{~g} / \mathrm{h}$ and $45 \mathrm{~g} / \mathrm{h}, 90 \mathrm{~g} / \mathrm{h}$ and $135 \mathrm{~g} / \mathrm{h}$ trials, respectively. Only the $90 \mathrm{~g} / \mathrm{h}$ trial significantly enhanced performance relative to placebo $(\mathrm{p}<0.05)$. There was no significant interaction between carbohydrate dose and GI distress, but there was a significant interaction between carbohydrate dose and blood glucose as well as estimated rates of carbohydrate and fat oxidation during steady state exercise. The results from this study support current carbohydrate intake guidelines and provide preliminary evidence for a lack of GI symptoms even at high feeding rates with a novel sport drink formulation.

\section{The Effects of Beta-Alanine Supplementation in Combination With Resistance Training on Fat-Free Mass}

M Macuh ${ }^{1}$, and A Anton-Solanas ${ }^{1}$

${ }^{1}$ Faculty of Sport, Health and Applied Science, St Mary's University, England
Beta-alanine is taken up and used by skeletal muscle to form carnosine, a potent buffer of hydrogen ions which has been shown to improve exercise performance. While there have been many studies done on the effects of beta-alanine on aerobic and anaerobic performance, research is lacking on the effects of beta-alanine on fat-free mass while following a resistance training intervention. The aim of this randomised controlled trial was to investigate the effects of beta-alanine supplementation in conjunction with resistance training on fat-free mass changes. Sample size calculation $(\alpha$-error $=0.95$; power $=80 \%)$ determined that fourteen participants were required for this study. Therefore, fourteen young males (age $23 \pm 3$ years) were randomly assigned to either the beta-alanine (BAG; $4.8 \mathrm{~g}$ betaalanine per day) or placebo group (PLG; $4.8 \mathrm{~g}$ maltodextrin per day) and underwent a resistance training program (4 days $\mathrm{x}$ week(-1)) lasting four weeks and consisting of 8-12 repetition maximum per set with 1.52.0-minute breaks between sets. This study followed the same resistance training and dietary intervention as Hoffman et al (MSSE 2008, 29(12), 952-958). Participants' nutritional intake was assessed via 3-day food diaries before and after intervention. Changes in fat-free mass were assessed using air displacement plethysmography before and after intervention. There was no significant effect of beta-alanine supplementation on fat-free mass $(+2.1 \%$ for BAG vs. $+1.6 \%$ for PLG; $\mathrm{p}=0.843)$, fat mass $(-2.1 \%$ for BAG vs. $-1.6 \%$ for PLG; $\mathrm{p}=0.984)$, body mass $(-0.9 \%$ for BAG vs. $-0.1 \%$ for PLG; $\mathrm{p}=0.378)$ or BMI $(-0.3 \%$ for BAG vs. $-0.2 \%$ for PLG; $p=0.971)$. There was no difference in energy intake, energy intake per $\mathrm{kg}$ of body weight, daily protein, carbohydrate or fat intake between and within subjects from before to after intervention indicating both groups had a similar diet throughout the study. In conclusion, despite observing a bigger change in fat-free mass in the BAG, the results did not reach statistical significance.

\section{An Interactive Database to Quantitatively Assess the Impact of Medication on Nutrient Status}

\section{Wakeman ${ }^{1}$}

${ }^{1}$ University of Sunderland, Edinburgh Building, City Campus, Chester Road, Sunderland, UK

The use of medication amongst sportsmen and women is commonplace, to both control and manage chronic conditions such as asthma which might impact upon performance and also aid recovery from injury. Typically around $85 \%$ of Paralympic and $74 \%$ of Olympic athletes have used one or more physician-prescribed medications within a 12 month period. There is a general lack of awareness that medications can negatively impact upon nutritional status especially when taken long term or used concurrently. This is mainly because the relevant literature is disparate and poorly collated. An evidence-based, personalised nutrition database has now been developed that assesses the potential impact of medication on nutritional status and the microbiome. Based upon inputted demographic parameters, dietary consumption and preferences such as vegetarianism or veganism, together with use and duration of treatment of any medication/s, it uses an algorithm to determine the likely effects these might have on nutritional status. The algorithm has been developed by a pharmacist, a GP and a dietician. The medication database is a comprehensive collection of over 3000 drugs available in UK, by brand and generic name. Any likely detrimental effects of single or multiple drugs are aggregated, and together with the data collected from the diet and lifestyle inputs, a summary score for any outcome on 28 separate vitamins, minerals, fatty acids, quasi vitamins, and microbiome status is computed. Almost 4000 interactions affecting nutritional status have been identified. A summary of findings is reported, with a hyperlink to the abstract of any clinical study where a relevant observation/s was reported, thereby enabling validation of any conclusions that have been reached. These are derived from in excess of 
1000 collated references. Potential minor and moderate negative impacts resulting from inputs are advised to be corrected firstly by diet, and any major potential deficiencies by additional supplementation wherever necessary. This development provides practitioners operating in the field the opportunity to access a tool that complements their clinical skills and contributes to optimise the nutritional status of their clients by delivering evidence-based recommendations derived from a systematic review of the scientific literature.

\section{Timing of Dietary Intake of British Army Officer Cadets in Camp and Field Training Environments}

VE Edwards ${ }^{1}$, SL Wardle ${ }^{2}$, SD Myers ${ }^{1}$, AG Siddall ${ }^{1}$, S Needham-Beck $^{1}$, SD Powell ${ }^{1}$, S Jackson ${ }^{2}$, JP Greeves ${ }^{2}$, and SD Blacker ${ }^{1}$

${ }^{1}$ Occupational Performance Research Group, University of Chichester, UK

${ }^{2}$ Army Personnel Research Capability, Army HQ, UK

The timing of dietary intake after exercise affects the adaptive response to physical training. During military training, personnel undertake a variety of concurrent and repeated strenuous activities over multiple days and eat only when time permits, making the quantification and optimisation of dietary intake challenging. The aim of this study was to quantify the timing of Officer Cadets' (OC) dietary intake in camp (CAMP) and field exercise (FEX) training environments during British Army Officer training. Twenty OCs (mean \pm SD: age $23 \pm 2$ years, height $1.74 \pm 0.08 \mathrm{~m}$, body mass 76.8 $\pm 9.5 \mathrm{~kg}$ ) volunteered for the study, 20 participated in CAMP and 17 on FEX. The CAMP dietary intake was measured by researcher-led dietary weighing of main meals alongside food diaries for 10 days and, the FEX, through weighed operational ration pack (ORP) discards for 6 days and analysed using nutritional software (Nutritics, Nutritics LTD, Ireland). Dietary intake timing was recorded by researchers in the cookhouse, by participants on a food diary or on the ORP item, and split into seven time points (pre-breakfast, breakfast, mid-morning, lunch, mid-afternoon, dinner and evening). Total intake between conditions were compared with T-tests and intake over time within conditions with a one-way independent analysis of variance (ANOVA). Total average energy intake (TEI) (CAMP; $3392 \pm 724$ vs. EX; $\left.2697 \pm 837 \mathrm{kcal} \cdot \mathrm{day}^{-1}\right)$, carbohydrate $(\mathrm{CHO})$ (CAMP; $412 \pm 101 \mathrm{vs.} \mathrm{EX;} 353 \pm 111 \mathrm{~g} \cdot \mathrm{day}^{-1}$ ) and protein (PRO) (CAMP; $128 \pm 29$ vs. FEX; $\left.79 \pm 24 \mathrm{~g} \cdot \mathrm{day}^{-1}\right)$ intake were greater during CAMP than FEX $(\mathrm{p}<0.05)$. For CAMP, TEI, CHO and PRO intake were greater for core meals than for snack periods $(\mathrm{p}<0.05)$, but no difference was seen in FEX. Whilst individual CAMP data reflected the mean data, the FEX intake pattern was highly variable, resulting in similar meal values across core meals and snack periods. In conclusion, OCs appear to eat sporadically, and when time permits during field exercise training, therefore the timing of intake may not be optimal for recovery during periods of continued work. Future research should examine whether optimising the timing of dietary intake around physical activity will enhance training adaptations and recovery during military training.

\section{The Impact of Hydration Status on Recovery in a Soccer Team}

\section{G. Vici ${ }^{1}$, L. Cesanelli ${ }^{1}$, L. Belli ${ }^{1}$, G. Vastano ${ }^{1}$, and V. Polzonetti ${ }^{1}$}

${ }^{1}$ Nutricam: Food and Health, School of Biosciences and Veterinary Medicine - University of Camerino, Italy

Many factors are shown to influence an athlete's performance such as good technical abilities, good mental skills and adequate body composition. In particular, good hydration status is critical during and after training sessions and competition events. As exercise performance and postexercise recovery can be significantly impaired when hydration status is not adequate, the aim of this study was to evaluate the impact of hydration status on recovery indexes on professional athletes. Twenty-eight professional soccer players, aged between 18 and $35 \mathrm{y}$, were subjects for the study. Body composition and in particular hydration status were assessed trough Bioelectrical Impedance Test (BIA) using single-frequency bioimpedance (BIA AKERN101). Recovery was analyzed using a specific test (GQR-TIA) where a scale from 0 to 10 indicates from "need absolute rest, no recovery" to "ready for the competition, total recovery". Statistical analysis was carried on using SPSS ${ }^{\circledR}$. Correlation coefficients were calculated to assess the relationship between variables. Significance level was set at $p \leq 0.05$. The analysis of body composition and recovery revealed that there is a statistically significant correlation between hydration status and recovery taking into account age. Indeed, it was possible to observe that in dehydrated subjects, as the age increases, test results decrease with a statistically significant correlation coefficient of -0.888 while in hydrated subject the correlation was found to be weaker, around an half, with a statistical significant correlation coefficient of -0.418 . High-intensity exercise in athletes and regularly active individuals typically results in dehydration that can affect physical performance and mental capacity. Moreover, it is well known that recovery after exercise is dependent on several factors i.e. age and body composition. From this study, it was possible to observe as dehydration has an important impact on recovery after exercise above all in relation to age. Indeed, as age increases, recovery index decreases twice faster in dehydrated subjects than in euhydrated ones. So, hydration status and above all specific rehydration strategies resulted to be of fundamental importance both to achieve a better performance and also to better recover after exercise or competition.

\section{Heart Rate Response to Pre-Exercise Meal: A Case Study}

\section{Cesanelli ${ }^{1}$, G. Vici ${ }^{1}$, L. Belli ${ }^{1}$, R. Ceci ${ }^{1}$, and V. Polzonetti ${ }^{1}$}

${ }^{1}$ Nutricam: Food and Health, School of Biosciences and Veterinary Medicine - University of Camerino, Italy

Pre-exercise meal is fundamental to support athletes to achieve training adaptation and maximize performance. Different strategies have been studied to improve performance or to cope with athlete's special needs. In parallel, it has been reported how food consumption may have a profound effect on body's metabolic rate and oxygen consumption (Specific Dynamic Action; SDA of food). Increased oxygen consumption by SDA of food is reflected by an elevated resting heart rate. To date, few studies have investigated the effect of SDA of food consumed previous to exercise on heart rate variability during exercise. Therefore, this pilot study aimed to evaluate the impact of different pre-exercise nutritional strategies on heart rate (bpm) during exercise at a given power (W) intensity. One amateur healthy cyclist aged 26 voluntarily participated to the study. Functional Threshold Power (FTP) and Functional Threshold Heart Rate (FTHR) tests were performed at the beginning of the study to assess intensity zones. Power output (W) was measured through a crankset power meter (Stages Cycling, LLC 2018), heart rate (bpm) and training data were acquired by a cyclocomputer (Bryton Rider 530). The subject performed 12 road test sessions, in which intensity intervals in terms of $\mathrm{W}$ were previously established. The tests were performed in duplicate in fasting conditions ( 8 hours); after Coffee consumption ( $80 \mathrm{mg}$ caffeine); after Very Low Carb High Fat meal; Low Carb meal; High Carb Low Fat meal and Equilibrated meal consumption. Heart rate data acquired from the tests were then compared with those expected (FTHR and FTP tests). Average heart rate during fasted trainings resulted lower $(-3.9 \mathrm{bpm})$ than expected while during fasted training with coffee greater $(+4.4 \mathrm{bpm})$ than expected. After very low carb high fat meal average training heart rate was lower $(-2.3 \mathrm{bpm})$ than expected on the contrary after high carbs meal was greater (+3.9 bpm). Low carb meal and equilibrate meal produced the lower 
deviations $(+1.9 \mathrm{bpm}$ and $+1.3 \mathrm{bpm}$ respectively). Our data suggest that even with a limited number of tests, deviations from expected values in terms of heart rate (bpm) are observable. This pilot study seems to suggest how pre-exercise meal composition could represent another considerable variable able to influence training heart rate output.

\section{Sleep Efficiency in a Cohort of Elite Level Soccer players}

T Simper ${ }^{1}$, T Lynn, S Bowles ${ }^{1}$, L Taylor ${ }^{2}$, and L Walton-Taylor ${ }^{1}$

${ }^{1}$ Sheffield Hallam University, UK

${ }^{2}$ Robert Gordon University, UK

We recorded sleep parameters via wrist worn accelerometers (GENEActiv) for 7 days in 14 elite level male soccer players in the middle of the 2016/17 football season. For comparison, we also collected 7-days of sleep data from 14 similar aged male, non-obese, physically active undergraduate University students, with no reported sleep problems. We sought to combine sleep duration and efficiency to rate sleep as either: 'good' 'average' or 'poor'. Individuals were classified as having good sleep quality based on an efficiency $\geq 85 \%$, and duration of $\geq 420 \mathrm{~min}$. Poor sleep quality was based on an efficiency $\leq 75 \%$ or duration $\leq 360 \mathrm{~min}$. The remaining individuals were therefore classified as having average sleep quality. We also measured dietary intake levels via 7-day dietary inventory for both groups during the week of sleep analysis. The footballers and students returned 67 and 70 nights of sleep data respectively. The mean average sleep efficiency for the footballers was very similar to that of the students $(79 \% \mathrm{v} 79 \% ; \mathrm{P}=0.67)$. In this group of academy level football players only $21 \%$ of the sample recorded sleep data would qualify as 'good' with $42 \%$ as 'average' and $37 \%$ as poor. This compares to the students' sleep which was $20 \%, 37 \%$ and $43 \%$, for good, average and poor respectively. The data were assessed as not meeting parametric requirements and subsequently between-group comparisons were carried out using Mann-Whitney U tests for: total energy, carbohydrate, fat, protein, caffeine, tryptophan and alcohol intakes. These were all not significantly different, with the exception of protein which was higher in the football players $(\mathrm{P}=0.009)$. In conclusion, the two groups appear similar in terms of sleep quality. Football players, who are required to recover efficiently from exercise and have high levels of concentration, have high levels of 'poor' and 'average' sleep quality. Interventions, such as sleep hygiene protocols, may improve sleep quality in football players (and students). Given that the shift needed for most of the cohort to move into the 'good' category is small in percentage terms, a relatively mild intervention may help football players make that shift.

Deviation in Predicted and Calculated Resting Metabolic Rates (RMR): Closing the Gap. A Case Study From an Elite British Judoka

\section{Bloor $^{1}$, R Cloak ${ }^{2}$, and K Staddon ${ }^{1}$}

${ }^{1}$ British Judo, British Judo Association, Wolverhampton University, Walsall Campus, Walsall, UK

${ }^{2}$ Departments of Sport and Exercise Science, Wolverhampton University, Walsall Campus, Walsall, UK

Judo is an Olympic Combat Sport where intense competition schedules require frequent energy deficits to enable judokas to make weight. Daily energy deficits must then be increased to match total daily energy expenditure during non-weight making phases to maintain high energy availability and ensure optimal health and performance. Failure to do this can lead judokas to maintain a long-term energy restriction, which over time has shown to be accompanied by metabolic and endocrine adaptations that can prevent further weight loss in order to conserve energy. This may then lead to a cycle of continued energy deficit by either reducing energy intake further or increasing energy expenditure leading to chronic energy deficits and in some instances putting them on the spectrum for relative energy deficit (RED-S). Deviations in predicted and calculated resting metabolic rate (RMR) has been used to measure the suppression of RMR caused by such adaptations that occur in chronically energy deficient athletes. Lower than expected RMR's have been reported in elite female rowers and endurance athletes and correlates closely with low energy availability. This case study outlines the approach taken to help an elite female judoka showing symptoms of RED-S to increase energy intake to support performance and reduce the risks associated with frequent weight making.

The athlete in question exhibited a deviation between predicted and calculated RMR of $-31 \%$ (a deficit of $481 \mathrm{kcal}$ ). Over a 6 -month period daily energy intake was increased from $1621 \mathrm{kcal}$ to $2262 \mathrm{kcal}$. Carbohydrate intake increased from $5.3 \mathrm{~g} / \mathrm{kg} / \mathrm{bw}$ to $6.9 \mathrm{~g} / \mathrm{kg} / \mathrm{bw}$, protein $1.4 \mathrm{~g} / \mathrm{kg} /$ bw to $3.1 \mathrm{~g} / \mathrm{kg} / \mathrm{bw}$ and fat was $0.8 \mathrm{~g} / \mathrm{kg} / \mathrm{bw}$ throughout the recorded period. Bone density T-Score increased $(+2.4-+2.5)$, body fat $\%$ increased $(13.6 \%-14.4 \%)$ and lean mass decreased $(41.6 \mathrm{~kg}-40.6 \mathrm{~kg})$. Body mass and sum of 8 skinfold measurements remained consistent $(49.85 \pm 0.75 \mathrm{~kg}$ and $51.5 \pm 0.5 \mathrm{~mm}$ respectively). At the end of the monitored period RMR deviation reduced from $31 \%$ to $16 \%$, a deficit of $263 \mathrm{kcal}$. All subjective markers around wellbeing, energy and ability to perform improved and weight making were reported as easier. This case study demonstrates that gradual increase in energy intake may increase energy availability, enhancing both health and performance markers and safer weight making practices.

Effect of a 6-Month Exercise and Nutrition Intervention on Bone Strength and Quality of Life of Professional Jockeys in Hong Kong

\section{E. Poon ${ }^{1}$, J. O'Reilly ${ }^{1}$, and S. Wong ${ }^{1}$}

${ }^{1}$ Department of Sports Science and Physical Education, The Chinese University of Hong Kong, Hong Kong

Weight-making practices regularly engaged by horse-racing jockeys are suggested to impair physiological and mental health. Despite the unsatisfactory bone condition among jockeys, population-specific intervention programs to improve their bone health and nutritional habits are rare. The purpose of this study was to examine the effect of an exercise and nutritional intervention on the markers of bone health and quality of life of Hong Kong-based professional jockeys. 13 professional male jockeys (horse racing experience: $3-25$ y; mean age: $29 \pm 6$ y; BMI: $20.2 \pm 1.6 \mathrm{~kg}$ $\mathrm{m}-2$ ) and 13 gender-, age-, and BMI-matched controls (mean age: $27 \pm 6 \mathrm{y}$; BMI: $21.4 \pm 1.7 \mathrm{~kg} \mathrm{~m}-2$ ) were recruited. Both groups completed a range of assessments including: (i) Anthropometry via skinfold measurement; (ii) Bone mineral density at calcanei and forearms via Dual-energy X-ray absorptiometry (DEXA); (iii) Nutritional intake via 3-day food diary and validated food frequency questionnaire (FFQ); (iv) Quality of life (QOF) questionnaire before and after a 6-month period. The jockey group received a 6-month intervention which involves (i) regular nutritional consultation with individualised advice and; (ii) exercise training that focused on osteogenic stimulation (i.e. high-intensity plyometric exercise). Paired-sample t-tests were used to compare the changes within both groups before and after the 6-month period. The 6-month intervention led to significant increase in calcium intake (pre: 451 vs post: $900 \mathrm{mg} /$ day), left forearm bone strength (T-score pre: $-0.23 \pm 1.29$ vs post: $0.12 \pm 1.48$ ) and improvement in perceived physical quality of life (pre: $72 \pm 3$ vs post: $78 \pm 4$ ) in the jockey group. No significant difference was found for the control group in all variables $(\mathrm{p}>0.05)$. In conclusion, our results revealed that a 6-month intervention targeting improved nutrition and exercise habits can enhance bone strength and perceived physical quality of life of elite 
jockeys in Hong Kong. Long-term monitoring of bone density and bone health biomarker is warranted in future research.

\section{An Exploration of the Factors that Influence Ultra-Endurance Athletes' Food Choices During Competition}

\section{Blennerhassett ${ }^{1}$, LR Mc Naughton ${ }^{2,3}$, L Cronin $^{2}$, G Stone ${ }^{1}$, L Nelson ${ }^{2}$,} and SA Sparks ${ }^{2}$

${ }^{1}$ Faculty of Health and Social Care, Edge Hill University, Ormskirk, UK

${ }^{2}$ Sport Nutrition and Performance Research Group, Department of Sport and Physical Activity, Edge Hill University, Ormskirk, UK

${ }^{3}$ University of Johannesburg, South Africa

The reasons for suboptimal nutritional intake within ultra-endurance competitions are not fully understood. Therefore, the current programme of research discusses the findings from a series of studies that explored the factors that influence the competitive food choices of ultra-endurance athletes. Study 1 indicated that nutrition knowledge was not a strong predictor of food choice in these athletes. Study 2 used a mixed methods approach to explore the wider influences of food selection during ultraendurance competition. In study 2, 101 ultra-endurance athletes completed a bespoke food choice questionnaire (U-FCQ) using a 7-point Likert style rating scale $(1=$ extremely unimportant to $7=$ extremely important). A further 14 ultra-runners took part in semi-structured interviews. High levels of importance were attached to the avoidance of gastrointestinal symptoms (GIS) during the U-FCQ $(6.6 \pm 0.8)$ and the interviews ('bloating' 'nausea'). Similarly, the need for energy $(6.5 \pm 0.8)$ and to overcome logistical challenges (6.1-6.3) was evident in both data sets. In the interviews, participants referred to specific nutrients ('carbohydrate' 'sodium') and food properties ('portability' 'chewy') that were either favourable or detrimental to nutritional intake. As an example, chewy foods were difficult to ingest and compromised intake. Many athletes rated being 'nutritious' $(6.3 \pm 0.7)$ as highly important to their food choices, but few runners commented on this during the interviews. Strong influences absent from the U-FCQ included the avoidance of other physiological symptoms ('cramps' 'hunger') and the athlete's personal values. This included sensory preferences for specific tastes and the 'familiarity' of individual items. Conflict between studies likely reflects situation specific differences in food choice that are common in the literature, along with the scope of the data collection tools. The interviews enabled the researchers to explore the complexity of the food choice process, which was not possible using the U-FCQ. Particularly, how athletes balanced conflicting demands for fuel and cues to stop eating, especially in the latter stages of the race. This suggests that athletes would benefit from a race nutrition plan that is flexible and allows them to manage competing, dynamic nutritional drivers.

\section{The Impact of Exercise-Induced Dehydration on Systemic Endotoxin and Inflammatory Cytokine Profiles}

Z Huschtscha ${ }^{1}$, A Russo ${ }^{1}$, RMJ Snipe ${ }^{2}$, D Dixon ${ }^{3}$, V Camões-Costa ${ }^{1,4}$, and RJS Costa ${ }^{1}$

${ }^{1}$ Department of Nutrition Dietetics \& Food, Monash University, Australia

${ }^{2}$ Centre for Sport Research, Deakin University, Australia

${ }^{3}$ Department of Sports Science, Writtle University College, UK

${ }^{4}$ Health \& Aging Research Group, Swinburne University of Technology, Swinburne University of Technology, Australia

Compromised gastrointestinal integrity in response to exercise stress has been shown to increase translocation of the luminal bacterial content into systemic circulation. However, the magnitude to which exercise-induced dehydration impact systemic endotoxaemia and responsive cytokinaemia is less known. The study aimed to determine the impact of exerciseinduced dehydration on systemic endotoxin and cytokine profiles. Using a randomised cross-over design, male endurance runners $(\mathrm{n}=11)$ performed $2 \mathrm{~h}$ of running at $70 \% \dot{\mathrm{V}} \mathrm{O}_{2 \max }$ in $25^{\circ} \mathrm{C} \mathrm{T}$ amb and $46 \% \mathrm{RH}$. On one occasion participants were provided with water $(1689 \pm 542 \mathrm{ml}$; $11.0 \pm 3.5 \mathrm{ml} / \mathrm{kg}$ ) during exercise (euhydration: EUH; $0.6 \pm 0.6 \%$ body mass loss (BML), $\mathrm{P}_{\text {Osmol }} 291 \pm 13 \mathrm{mOsmol} / \mathrm{kg}$, and $\Delta \mathrm{P}_{\mathrm{V}}-1.0 \pm 5.0 \%$ ), and on another occasion participants were water restricted (dehydration: $\mathrm{DEH}$; $3.1 \pm 0.7 \% \mathrm{BML}, \mathrm{P}_{\mathrm{Osmol}} 306 \pm 11 \mathrm{mOsmol} / \mathrm{kg}$, and $\Delta \mathrm{P}_{\mathrm{V}}-5.8 \pm 3.7 \%$ ). Blood samples were collected pre- and post-exercise, and during recovery to determine leukocyte counts, neutrophil function, plasma bacterial endotoxin and inflammatory cytokine profiles. DEH did not result in greater exercise-induced leukocytosis (overall pre- to post-exercise: 5.9 to $\left.12.1 \times 10^{9} / \mathrm{L}\right)$ over EUH. Post-exercise depressed bacterially-stimulated neutrophil degranulation was observed on DEH (-36\%), whilst EUH prevented this depression $(+6 \%)$. DEH did not substantially increase systemic endotoxin profile (gram-negative bacterial endotoxin $+4 \mathrm{pg} / \mathrm{ml}$ and $\mathrm{sCD} 14-37 \mathrm{ng} / \mathrm{ml})$ over EUH $(+2 \mathrm{pg} / \mathrm{ml}$ and $+53 \mathrm{ng} / \mathrm{ml})$. A greater inflammatory profile was observed post-exercise on $\mathrm{DEH}(\Delta$ pre- to postexercise: IL-6 $+154 \%, p=.012 ; \mathrm{IL}-1 \beta+145 \%, \mathrm{p}=.091 ; \mathrm{TNF}-\alpha+56 \%$, $\mathrm{p}=.063 ; \mathrm{IL}-8+114 \%, \mathrm{p}=.012 ; \mathrm{IL}-10+526 \%, \mathrm{p}=.012 ; \mathrm{IL}-1 \mathrm{ra}+151 \%$, $\mathrm{p}=.012$ ), compared with EUH (IL-6 +124\%, $\mathrm{p}=.017$; IL-1 $\beta+22 \%$, $\mathrm{p}=.612 ; \mathrm{TNF}-\alpha+17 \%, \mathrm{p}=.310 ; \mathrm{IL}-8+52 \%, \mathrm{p}=.012 ; \mathrm{IL}-10+458 \%$, $\mathrm{p}=.012$; IL-1ra $+90 \%, \mathrm{p}=.012$ ); but there were no differences between groups ( $\mathrm{p}>.05)$. Unlike extreme endurance exercise (e.g., ultramarathon), with or without hypohydration, DEH does not substantially instigate systemic endotoxaemia above the body's clearance capacity. However, DEH does depress neutrophil functional responses and creates a modestly higher systemic inflammatory response, compared to EUH.

\section{New Zealand Blackcurrant Powder Reduces a Human Body Odour Component in Middle-Aged and Older Adults}

MET Willems ${ }^{1}$, M Todaka $^{2}$, MD Cook ${ }^{3}$, and Y Sekine ${ }^{2}$

${ }^{1}$ Institute of Sport, University of Chichester, UK

${ }^{2}$ Graduate School of Science, Tokai University, Japan

${ }^{3}$ Institute of Sport and Exercise Science, University of Worchester, UK

Nutrition and exercise can affect oxidative stress. Age-induced body odor is associated with emission of the skin gases 2-nonenal and diacetyl. Diacetyl is produced from L-lactate in sweat by bacterial activity. Higher emission of 2-nonenal in older people results from oxidative stress-induced lipid peroxidation of fatty acids in sebaceous glands by declined antioxidant defences. Blackcurrant has antioxidant effects. We examined whether New Zealand blackcurrant (NZBC) powder decreased the emission of 2 -nonenal in older adults. Fourteen middle aged and older adults ( 9 males, age: $55 \pm 5$ y, mean $\pm S D$, range $49-64$ y) volunteered. Participants consumed NZBC powder for 7 days (Sujon Berries, Nelson, New Zealand,

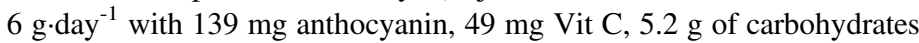
and total phenolic content of $272 \mathrm{mg}$ per serving). Two hours after the last intake, a passive flux sampler was applied to the skin in the base of the neck for one hour. Participants did not use hot water or soap for $48 \mathrm{hrs}$ on the skin sampling location. The sampler consists of a polypropylene screw cap with trapping media (MonoTrap ${ }^{\circledR}$, DCC18, GL Science, Japan) and gas chromatography-mass spectrometry was used for media analysis of 2-nonenal and diacetyl. Dietary anthocyanin intake was quantified using a food frequency questionnaire. No effect was observed for diacetyl (baseline: $2.7 \pm 1.7$, NZBC powder: $3.2 \pm 1.5 \mathrm{ng} \cdot \mathrm{cm}^{-2} \cdot \mathrm{hr}^{-1}$ ). With NZBC powder, 2-nonenal was reduced by $28 \%$ (baseline: $4.66 \pm 3.44$, NZBC powder: $2.4 \pm 1.5 \mathrm{ng} \cdot \mathrm{cm}^{-2} \cdot \mathrm{h}^{-1}$, two-tailed Mann-Whitney, $\mathrm{P}=0.01$ ) with six participants more than $69 \%$. The two participants with highest baseline 
2-nonenal, i.e. 9.4 and $13.8 \mathrm{ng} \cdot \mathrm{cm}^{-2} \cdot \mathrm{h}^{-1}$, showed reductions of $80 \%$ and $82 \%$. Baseline 2-nonenal values were not significantly correlated with dietary anthocyanin intake. The $\%$ decreases in 2-nonenal with intake of NZBC powder were not significantly correlated with baseline values. It is concluded that 7-day intake of NZBC powder is able to reduce body odor in older adults. The reduction in 2-nonenal by intake of NZBC powder was not associated with normal dietary intake of anthocyanins or baseline 2nonenal emissions.

Acknowledgement: We thank Sujon Berries (Nelson, New Zealand) for supplementation and Blackcurrant New Zealand Inc. for conference attendance.

\section{Almonds as an Energy Source in Endurance Exercise}

L Esquius $^{1,2}$, O Niño ${ }^{1,3}$, Alamo $^{1}$, and C Javierre ${ }^{1}$

${ }^{1}$ Department of Physiological Sciences, Medical School, University of Barcelona, Barcelona, Spain

${ }^{2}$ FoodLab, Faculty of Health Sciences, Universitat Oberta de Catalunya, Barcelona, Spain

${ }^{3}$ Faculty of Sports Science and Physical Education, University of Cundinamarca, Colombia
Several studies have investigated the effects of fat feeding before exercise on the subsequent rates of substrate oxidation and exercise performance. Some results revealed that unsaturated fatty acids supplementation tended to slightly increase fat oxidation. However, these changes were not reflected in $\mathrm{VO}_{2 \max }$ or in other performance and physiological parameters. We selected almonds as a source of fatty acids to make an acute fat supplementation. Almonds, by weight, are about 50\% lipids, mostly monounsaturated fatty acids, which could be an energetic substrate. We made an original almond product (almond supplement) and investigated its effects on nonesterified fatty acids (NEFA) values and exercise performance. The almond supplement's main ingredients were almonds and the placebo supplement's main ingredient was bread. Five male and trained subjects went through two separate cycling effort test sessions separated by 7-day interval. The supplements were taken two hours before the test. Several blood samples were collected before, during and after testing for biochemical measurements. The results showed a NEFA increase of $0.09 \mathrm{mg} \cdot \mathrm{dL}-1 \quad(95 \%$ $\mathrm{CI}=0.05-0.14)(\mathrm{p}<0.0001)$ during the test in those taking the almond supplement in comparison to those taking the placebo. Almonds supplementation enhanced performance in comparison to placebo (almonds supplementation $5389 \pm 1795 \mathrm{w}$ vs. placebo $4470 \pm 2053 \mathrm{w}, \mathrm{p}=0.043$ ). This supplementation rich in fatty acids did not show gastrointestinal disturbances. Our study suggests that an acute almond supplementation taken two hours before exercise can improve performance in trained subjects. 